\title{
The use of the Open-Loop Onset Point (OLOP) to predict rotorcraft pilot-induced oscillations
}

\author{
Michael Jones ${ }^{1}$
}

Received: 21 May 2019 / Revised: 18 October 2019 / Accepted: 31 March 2020 / Published online: 20 April 2020

(c) The Author(s) 2020

\begin{abstract}
The Open-Loop Onset Point (OLOP) criterion has, for many years, been successfully used as a method to predict quasi-nonlinear pilot-induced oscillations (PIOs) for fixed-wing aircraft. Only limited research has been conducted using the criterion for prediction of PIOs occurring in rotorcraft. This paper details a study to extend the application of OLOP to rotorcraft, using the combination of control inputs appropriate for the task and a suitable pilot model. Results are compared between pilot subjective opinion and OLOP predictions, from tests performed in a ground-based simulation facility. Using 'taskspecific' application of OLOP, results obtained in the investigation are encouraging, whereby the objective predictions reflect subjective pilot assessment. From results obtained, a modified boundary is presented.
\end{abstract}

Keywords Rotorcraft · Pilot-induced oscillations · OLOP

\section{List of symbols}

$K_{\mathrm{e}}$

$U_{\mathrm{M}}$

$Y_{\mathrm{c}}$

$Y_{\mathrm{e}}$

$Y_{\mathrm{p}}$

$Y_{\mathrm{NM}}$

$Y_{\mathrm{PF}}$

$Y_{\mathrm{v}}$

$Y_{\mathrm{FS}}$

$Y_{\mathrm{VF}}$

$p, q$

$\delta_{\text {act }}$

$\delta_{\mathrm{c}}$

$\delta_{\mathrm{F}}$

$\tau_{\mathrm{p} \theta}$

$\phi, \theta$

$\theta_{\text {com }}$

$\omega_{\mathrm{BW}}$

$\omega_{\mathrm{c}}$

$\omega_{\mathrm{OLOP}}$

Michael Jones

michael.jones@dlr.de

1 Rotorcraft Department, German Aerospace Center, Lilienthalplatz 7, 38108 Brunswick, Germany

Visual compensation error

\author{
Subscripts \\ LAT \\ Lateral axis \\ LON \\ lat0 \\ Longitudinal axis \\ lat60 \\ lon0 \\ Lateral, hover \\ Lateral, 60 kts forward flight \\ Longitudinal, hover
}

Pilot compensation

Bare airframe vehicle transfer function

Abbreviations

$\mathrm{AC}(\mathrm{G}) \quad$ Attitude command (good HQs)

$\mathrm{AC}(\mathrm{P}) \quad$ Attitude command (poor HQs)

ACT/FHS Active control technology/flying helicop-

ter simulator

ADS Aeronautical design standard

APC(R) Adverse pilot coupling (rating)

ARISTOTEL Aircraft and rotorcraft pilot couplings:

tools and techniques for alleviation and detection

AVES Air vehicle simulator

Cat. II Category II pilot-induced oscillations

CONDUIT Control designer's unified interface soft-

ware package

DLR Deutsches Zentrum fuer Luft und Raumfahrt (German Aerospace Center)

FCS Flight control system

$\mathrm{HQ}(\mathrm{R}) \quad$ Handling qualities (rating)

HQSF Handling qualities sensitivity function

MTE Mission task element

OLOP Open-Loop Onset Point

$\mathrm{PIO}(\mathrm{R}) \quad$ Pilot-induced oscillation (rating) 


$\begin{array}{ll}\text { PVS } & \text { Pilot-vehicle system } \\ \text { RC } & \text { Rate command } \\ \text { RL } & \text { Rate limit } \\ \text { RLE } & \text { Rate limiting element } \\ \text { RPC } & \text { Rotorcraft pilot coupling } \\ \text { TS } & \text { Task specific } \\ \text { VMS } & \text { Vertical motion simulator }\end{array}$

\section{Introduction}

The occurrence of pilot-induced oscillations (PIOs) due to rate limiting in operational rotorcraft is rare. However, when so-called 'Category II' PIOs (Cat. II, quasi-non linear behaviour [1]) occur, it may lead to catastrophic failure (destroying equipment and causing loss of life [2]). Typically, these PIOs will be caused by non-linear elements in the control system, such as rate and saturation limits. Rate limits (RLs) in particular have caused many of the most dramatic PIOs found in the literature [1]. Rate limits cause non-linear system response when the command magnitude and frequency exceed the 'saturation point'. This results in an additional system delay, which can be characterised by the magnitude and frequency of input [3]. A range of RLs are used in rotorcraft control systems to prevent excessive rate of change (e.g. of surface or control element). Whilst most RLs are used to prevent abnormal response, other RLs may be used to tune control parameters or suppress vehicle response modes. A typical use of RLs is to restrict the rate of actuator travel, to protect hardware and software limits. Examples include rate or position limiters implemented into the FCS to protect systems from physical damage [4]. These RLs are activated when large or high frequency inputs are applied to the actuator. Continued 'activation' of these limits will lead to sustained saturation, and Cat. II PIOs. In normal operation, unexpectedly large- or high-frequency inputs may occur due to certain task conditions, for example, during tight control (high gain) tracking tasks, during operations in adverse weather conditions or due to the pilot experience (i.e. overcontrol of the vehicle by students during training flights [5]). Another potential source of Cat. II PIOs is control system failures, which cause changes in the pilot-vehicle system response [6,7]. Triggering this type of PIO is a particular concern during the development of novel rotorcraft configurations and experimental flight testing. In these circumstances, many flight control systems (FCS) are tested (with a pilot-in-the-loop) for the first time. There is a significant risk that the vehicle RLs may be encountered. This has been found in previous investigations [8].

For rotorcraft, this risk exists due to the lack of a unified method to observe the influence of rate limiting and the potential for Cat. II PIOs. Current Handling Qualities (HQ) guidelines for rotorcraft, Aeronautical Design Standard 33
(ADS-33 [9]), offer a range of predictive HQ metrics to determine if the vehicle will be prone to linear-type PIOs (i.e. Category I type events). For example, using bandwidthphase delay criterion, it is possible to ensure that the vehicle meets Level $1 \mathrm{HQ}$ requirements and is robust to this type of PIO (under normal operational conditions).

ADS-33 criteria are also used to develop novel control systems, using optimisation programs such as CONDUIT [10]. These tools make use of HQ and stability criteria to tune the response of the vehicle. Control system parameters are optimised to ensure that the response of the vehicle is within desired objective, hard, and soft constraints. The application of these criteria can lead to very agile control systems. However, the application of the criteria from ADS33 do not account for the presence of rate-limiting elements (RLE) within the control systems. For this reason, they must be separately considered. For this purpose, the OpenLoop Onset Point (OLOP) criterion can be applied [11]. The OLOP criterion was specifically designed to predict the incipience of PIO caused by rate limiting and is based on fixed-wing vehicle models and flight test data. Application to rotorcraft has been limited to a number of studies, discussed in the following section.

In this paper, the specific application of the OLOP criterion to rotorcraft is investigated and extensions to the methodology are proposed. The paper proceeds as follows. First, for completeness, the 'classical OLOP method' is introduced. The extensions to the method proposed in this research are introduced. Second, the pilot model used for the analysis, the Hess structural pilot model, is explained and parameters of the model are introduced. Third, the setup for the pilot-in-the-loop investigation is explained, including the tasks, pilots, and simulation facility used in the investigation. Next, the OLOP predictions based on the FCS models and RLs used are shown. Following this, all results from the simulation campaign are presented alongside OLOP PIO predictions. Finally, conclusions from the work are presented.

\section{The open loop onset criterion}

This section describes the OLOP criterion and extensions made in this research for its application to rotorcraft.

\subsection{Original conception and application}

OLOP is a method specifically designed to determine the PIO susceptibility due to RLEs within the pilot-vehicle system (PVS). It was proposed by Duda [11] as a method specifically tailored for predicting Cat. II PIOs using linearised models of fixed-wing aircraft. Since its initial conception, over the past 20 years, it has been applied to a wide range of vehicles. The method is based upon the use of describing 
functions to approximate RLEs in the system. Normal RLEs can be described by the input amplitude and a so-called open onset frequency $\left(\omega_{\mathrm{OLOP}}\right)$. This is the frequency where the RLE is 'activated' for the first time.

Research in Ref. [11] showed that the activation of RLEs leads to a rapid increase in phase distortion. This is referred to as a 'phase-jump'. Observing the frequency and dynamics at the point where the phase jump occurs allows one to determine whether the activation of RLEs has the potential to cause Cat. II PIOs. Using an extensive database of fixedwing Cat. II PIO events, Duda defined a boundary to determine PIO susceptibility using a Nichols chart. This boundary is shown in results presented in this paper.

OLOP has a number of advantages when investigating rate limiting. First, it can be applied during linear analysis of control systems, typically employed during the development phase. Second, it can be applied to RLEs at different points in the FCS. RLEs both in the forward and feedback control paths can be analysed. Third, the criterion accounts for changes in pilot-vehicle dynamics due to the FCS.

\subsection{Application to rotorcraft}

A number of studies, including those detailed in Refs. [4, $8,12-16]$, have used OLOP for analysis with varying success. Generally, OLOP has been used only when PIOs were encountered during research campaigns. In Ref. [8], it was recognised that using ADS-33 criteria and linearised models could lead to very high system bandwidth, which was initially found to tune the roll and pitch axis command model bandwidths to meet Level 1 requirements. When testing a $\mathrm{H}-53$ model with these bandwidths for the first time in the vertical motion simulator (VMS), RLEs of the actuator models were reached, and the pilots had difficulty flying the system. Divergent PIOs occurred at the end of one test run and pilots commented that the vehicle was very PIO sensitive, uncontrollable and quick to diverge. Post analysis of the system was conducted using OLOP and the susceptibility of the system was confirmed. Here the legitimacy of the method for application to rotorcraft was confirmed.

The method is also included in CONDUIT to ensure that the non-linearities in system actuators and limits are accounted for when using linearised methods to determine control system parameters. In Ref. [12], it was recommended that OLOP is applied to rotorcraft without a pilot model as most rotorcraft are bare-airframe unstable. The authors state that very conservative estimates of rate limiting onset are obtained when using a pilot model and, when enforced, severely limits performance. Therefore, OLOP is used without a pilot model so that the results are less conservative. Duda stated that this method is acceptable for RLE in the FCS and not for RLE at the pilot input [11].
In Refs. [13, 14], the method was investigated for prediction of Cat. II PIOs for rotorcraft during forward flight. The results from the application of OLOP were compared with subjective pilot assessment obtained in simulation using a roll tracking task. Pilot ratings suggested a disagreement between OLOP boundaries proposed by Duda and PIO susceptibility. As a result of this research, a new less conservative OLOP boundary was proposed. The research, however, was based on a very limited test database.

Extension to this work was conducted during the ARISTOTEL project (Aircraft and Rotorcraft Pilot Couplings: Tools and Techniques for Alleviation and Detection). In this research, further investigations using OLOP were undertaken, using two research simulators. Furthermore, a number of different low-speed and forward flight tasks were used to collect pilot subjective assessment and objective data. In this research, the conservative nature of the OLOP boundary was confirmed $[4,15]$.

The application of OLOP criterion for various flight speeds was also investigated in Ref. [16]. In this research, results suggested that the use of the maximum control input led to conservative predictions, which did not reflect the pilot activity during piloted ADS-33 mission task elements (MTEs) or during normal operation of the vehicle. As a result, it was proposed that OLOP is applied using realistic inceptor deflection (normal pilot actions).

Due to the conservative nature of the boundary for rotorcraft investigations, no standardised method for the application of OLOP currently exists. Both changes to the boundary and the process have previously been proposed. The following section outlines the extensions and modifications to the OLOP process, which are proposed in this research, to increase its suitability for application to rotorcraft predictions.

\subsection{Method of application}

To apply the OLOP criterion, a linear model of the aircraft, the location of the relevant RLE, and information regarding the maximum control deflections are required [17]. The application is performed using the following four steps:

1. Determine the open-loop transfer function between pilot input and actuator input.

2. Determine the open-loop onset point ( $\left.\omega_{\mathrm{OLOP}}\right)$.

3. Select a pilot model.

4. Determine the transfer function of the PVS by breaking the loop at the RLE.

In Step 1, the transfer function between pilot input and vehicle input is determined with respect to frequency. In Step 2, $\omega_{\mathrm{OLOP}}$ is determined. To simulate the worst-case scenario, the transfer function obtained in Step 1 is multiplied by the 
maximum control input. This transfer function is compared to the transfer function of the RLE, performed using information contained in Fig. 1. The (lowest) frequency where the gain of both the transfer function of the control input and the RLE are equal is determined as the $\omega_{\mathrm{OLOP}}$. This is the frequency where the RLE will be 'activated'. The example in Fig. 1 shows the calculation of $\omega_{\text {OLOP }}$ for two RLs: $35 \% / s$ and $17 \% / s$. The determined $\omega_{\mathrm{OLOP}}$ is independent from the pilot model used.

To determine whether the RLE will lead to PIOs, a pilot model is required. The model is found using the open-loop vehicle transfer function. Duda states that a pilot model should be employed, and should be tuned to give an openloop crossover phase between $-120^{\circ}$ and $-160^{\circ}$ [11]. Tuning the crossover phase can be used to vary the 'pilot gain'. A low gain pilot is represented by $\mathrm{a}-120^{\circ}$ phase crossover, whilst $-160^{\circ}$ represents a high-gain pilot.

Once the pilot model has been determined, the transfer function of the PVS is determined by 'breaking the loop' at the RLE. An example of this is shown in Fig. 2. In this case, limiting occurs at the actuator output. The resulting openloop transfer function is plotted using a Nichols chart along with $\omega_{\mathrm{OLOP}}$ found in Step 2. The OLOP boundary is used to determine whether the case is prone to PIO.

The method described above is referred to as "classical OLOP'. As the analysis is conducted by 'breaking the loop' at the RLE, the method is suitable for the analysis of RLEs

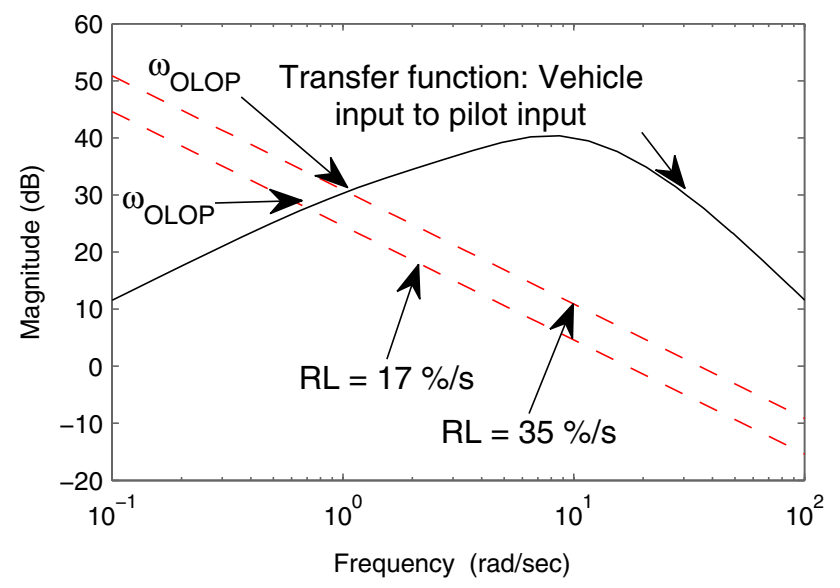

Fig. 1 Determining the OLOP using the transfer function between pilot input and vehicle control input in both the forward and feedback control channels. The use of the method for both cases has been extensively validated for fixed-wing aircraft [18]. In this research, the closed-loop model shown in Fig. 2 is used.

For the application of OLOP, models of the pilot, bare airframe vehicle $\left(Y_{\mathrm{c}}\right)$, force feel characteristics $\left(Y_{\mathrm{FS}}\right)$ and FCS are used. For the application of the 'classical OLOP' method, Duda suggested to use a pilot model represented by a pure gain (regardless of closed-loop system dynamics). As stated above, this pilot model is tuned so that the open-loop PVS leads to a crossover phase between $-120^{\circ}$ (low-gain pilot model) and $-160^{\circ}$ (high-gain pilot model). In this research, the RLE is used to break the closed-loop system between the FCS and $Y_{\mathrm{c}}$. This can be seen in Fig. 2 .

Figure 3 shows examples of results found using the "classical' application of OLOP. For these case, the high-gain pilot model is used (i.e. phase crossover of $-160^{\circ}$.). The results are displayed on a Nichols chart, showing the relationship between phase and amplitude. Two examples are shown: one PIO prone and one PIO robust case. Also shown is the OLOP boundary proposed by Duda [11]. As previously stated, if 'activated', the RLE will cause a 'phasejump'. The $\omega_{\mathrm{OLOP}}$ shows at which frequency this will occur. By plotting this point on the Nichols chart, both the phase and amplitude of the closed-loop model can be determined. Duda found that for cases where the onset-point occurred at low open-loop system gain, the increased phase distortion would not influence pilot control activity, has no destabilising effect and, therefore, does not lead to PIO. Conversely, when the onset-point occurs at higher open-loop system gain, a destabilising affect will occur, and PIOs would be experienced during attempted closed-loop control.

As shown in Fig. 3, $\omega_{\text {OLOP }}$ below the boundary will result in a PIO robust system. An $\omega_{\mathrm{OLOP}}$ above the boundary results in a PIO prone configuration. This information can be used to determine design parameters for the control system. Once the PIO susceptibility is known, a number of methods are applicable to reduce the potential for PIO. First, and obviously, a reduction in the rate limiting will lead to an increase in $\omega_{\mathrm{OLOP}}$ frequency. This will push the point further towards the PIO robust region. A second possibility is to decrease the pilot control gearing, to limit the amplitude of the openloop system at $\omega_{\text {OLOP }}$. Third, if the RLE and control gearing cannot be modified, efforts can be made to manipulate parameters in the FCS to reduce PIO susceptibility.

Fig. 2 Closed-loop system used in this research, example shown for pitch axis

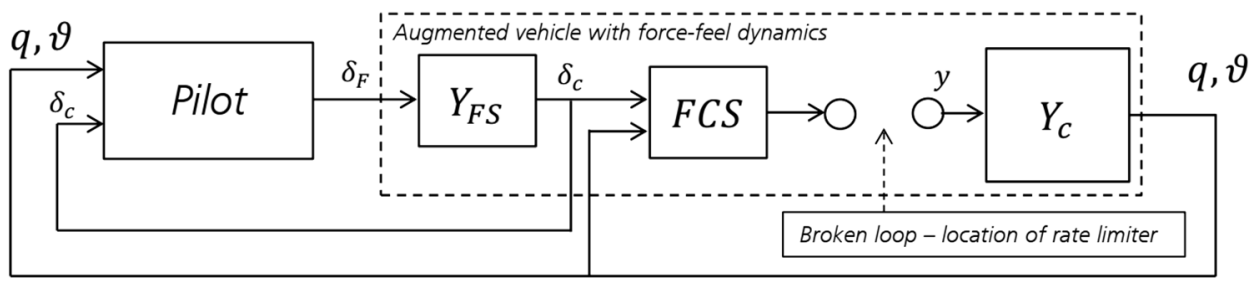




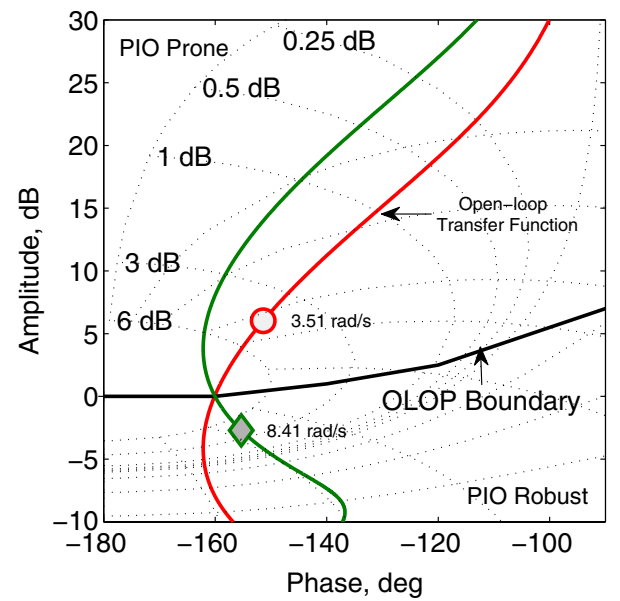

Fig. 3 Example of the application of 'classical OLOP'

\subsection{Extensions to OLOP}

Two extensions to the OLOP model are proposed in this research: the inclusion of a sophisticated pilot model and the use of task-specific control input magnitudes.

Most previous applications have used a simple gain pilot model (as used during the original conception). Tuning of the pilot model is conducted by tuning the model to achieve an specified open-loop phase margin at gain crossover (i.e. $0 \mathrm{~dB}$ ). The phase margin is the difference between the instability phase (i.e. $-180^{\circ}$ ) and the phase at crossover. The use of this model can lead to high crossover frequency, unrepresentative of the actual pilot dynamics. The model also does not include any considerations for the control dynamics or the delay during compensatory pilot control. In Ref. [19], OLOP was applied using a Neal-Smith pilot model. This model was tuned to replicate low- and high-gain pilot dynamics. The model was found to be suitable for use with the OLOP method.

In this research, OLOP analysis is performed using Hess' structural pilot model. This model has been selected as it has been previously shown to be suitable for rotorcraft closedloop task analysis [20, 21]. Furthermore, the model incorporates neuromuscular dynamics, control inceptor dynamics and vestibular feedback. All of these elements have been shown to influence the susceptibility of the vehicle to PIO $[4,6,15]$. With the use of the Hess model, detailed analysis of the influence of these elements can be conducted to assist in the design process. For example, tuning the control inceptor dynamics could be used to avoid PIOs rather than required modification to the FCS.

The second modification to the OLOP process is the use of appropriate control input magnitudes. It is believed that the conservative predictions found in previous research are due to the requirement to use maximum control input magnitude to determine $\omega_{\mathrm{OLOP}}$. In reality, the pilot would only provide inputs of this magnitude during an emergency or failure situation. Whilst application of OLOP using this control magnitude ensures that the vehicle is completely PIO free through the entire control envelope, this is unlikely to occur during testing. Typically, for normal rotorcraft operations, the pilot will use only $20-30 \%$ of the maximum control input. For trimmed control and normal operations, an additional safety margin is required. Not only is it unlikely that the pilot will reach full control displacement, it is also unlikely that oscillatory control input will be sustained at this magnitude. Therefore, for the application in this research, it is proposed to use command inputs expected for the defined tasks. This is achieved by determining an approximate/estimate of the required control deflections to complete the task. This can be performed either through initial pilot-in-the-loop tests or through the use of a model of the PVS dynamics. The required control input is then used in place of the maximum control input during 'Step 2' of the OLOP process.

Figure 4 shows the sensitivity of $\omega_{\mathrm{OLOP}}$ to the input size. For this example, $\omega_{\mathrm{OLOP}}$ has been calculated for pilot control input deflections between $( \pm) 10$ and 50\% maximum travel. $50 \%$ is considered to be maximum control input (i.e. oscillatory control with a maximum control range of $100 \%$ ). Numbers alongside each $\omega_{\mathrm{OLOP}}$ show the frequency in rad/s. As shown, for this example, with a pilot input above $30 \%$, the vehicle is predicted to be PIO prone. This dependency on inceptor input magnitude was also recognised in research detailed in Ref. [19], investigating the use of OLOP for fixed-wing aircraft. Here, the metric 'stick ratio' was proposed to improve OLOP predictions.

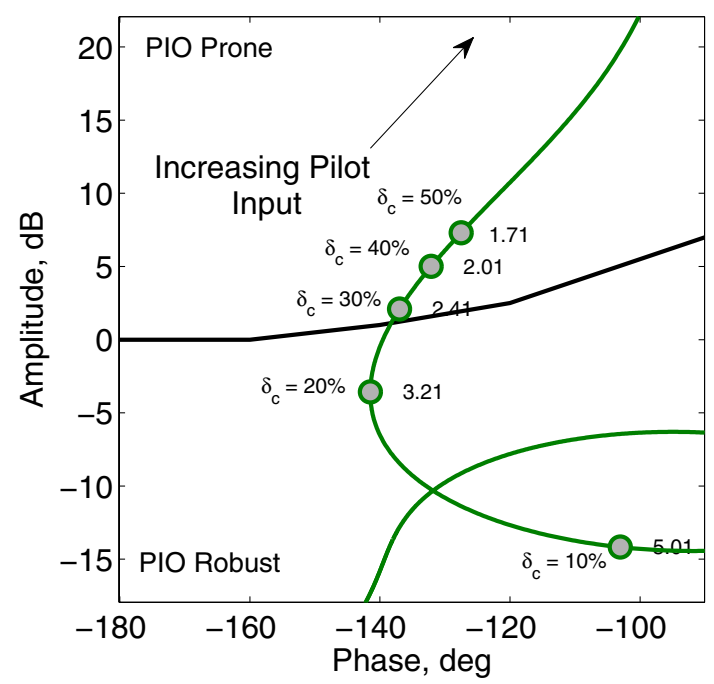

Fig. 4 OLOP sensitivity to pilot input magnitude 
The result from the modifications leads to a 'task specific' (TS) version of OLOP, whereby analysis is made for the specific situation where the vehicle is flown.

\section{Parametrising Hess' structural pilot model}

The Hess structural pilot model is shown in Fig. 5. This is in the form as presented in Ref. [20], as previously used for rotorcraft investigations. The model includes vestibular feedback, neuromuscular dynamics, visual error compensation and proprioceptive feedback. These elements of the model are discussed below. The Hess model requires parametrisation before it can be used. These parameters are dependent on the vehicle, pilot, and the 'simulation environment' dynamics.

\subsection{Pilot gain and crossover frequency}

In Ref. [20], a method is outlined to extract task-based crossover frequency. It is also stated that it is generally appropriate, for rotorcraft flying tasks, to select an open-loop crossover frequency $\left(\omega_{\mathrm{c}}\right)$ of $2 \mathrm{rad} / \mathrm{s}$. This generally specified crossover frequency is used in this research. The pilot gain is contained within the visual error compensation block is given by Eq. (1). To obtain an $\omega_{\mathrm{c}}$ of $2 \mathrm{rad} / \mathrm{s}$, the pilot gain $K_{\mathrm{e}}$ was tuned using an iterative loop.

$Y_{\mathrm{e}}=K_{\mathrm{e}} \mathrm{e}^{-0.2 \mathrm{~s}}$

\subsection{Form of proprioceptive feedback and neuromuscular dynamics}

As stated in Ref. [21], the form of the proprioceptive feedback is central to the model, and represents the pilots "internal model" of the dynamics. Its form is, therefore, dependent upon the vehicle dynamics, whereby the principles of the crossover model are observed [22];
$Y_{\mathrm{p}} Y_{\mathrm{v}}=\frac{\omega_{\mathrm{c}}}{s} \mathrm{e}^{-\tau_{\mathrm{e}} s}$,

where $Y_{\mathrm{p}}$ represents the pilot dynamics, and $Y_{\mathrm{v}}$ represents the vehicle dynamics (including FCS), and $\omega_{\mathrm{c}}$ is open-loop crossover frequency. The form of the proprioceptive feedback is therefore chosen so that, in the region of crossover, $Y_{\mathrm{PF}} \propto s Y_{\mathrm{v}}$. The magnitude of the pure gain is selected so that the closed-loop poles of the proprioceptive system have a minimum damping ratio of 0.15 . [20]. For this investigation, the form of the proprioceptive feedback was selected using the method stated in Ref. [20]. The form of the feedback was dependent on the FCS used. Neuromuscular dynamics were taken from Ref. [20] (Eq. 3).

$Y_{\mathrm{NM}}=\frac{10^{2}}{s^{2}+2(0.707) 10 s+10^{2}}$

\subsection{Vestibular feedback}

The Hess model allows the vestibular feedback to be modelled, whereby Eq. (4), a simplified gain of feedback of the angular rate is used. In this investigation, for pilot-inthe-loop tests, fixed-base simulation was used throughout. Therefore, $K_{\dot{m}}=0$.

$Y_{\mathrm{VF}}=s K \dot{m}$.

\subsection{Force feel feedback}

A significant advantage of the Hess structural model is the inclusion of force-feel characteristics. Using the proprioceptive feedback loop, the impact of the force-feel characteristics can be observed. For the investigation, an approximation of the force-feel characteristics was given by Eq. (5). The force-feel characteristics used in this investigation were previously used in research discussed in Ref. [23].
Fig. 5 Hess structural pilot model

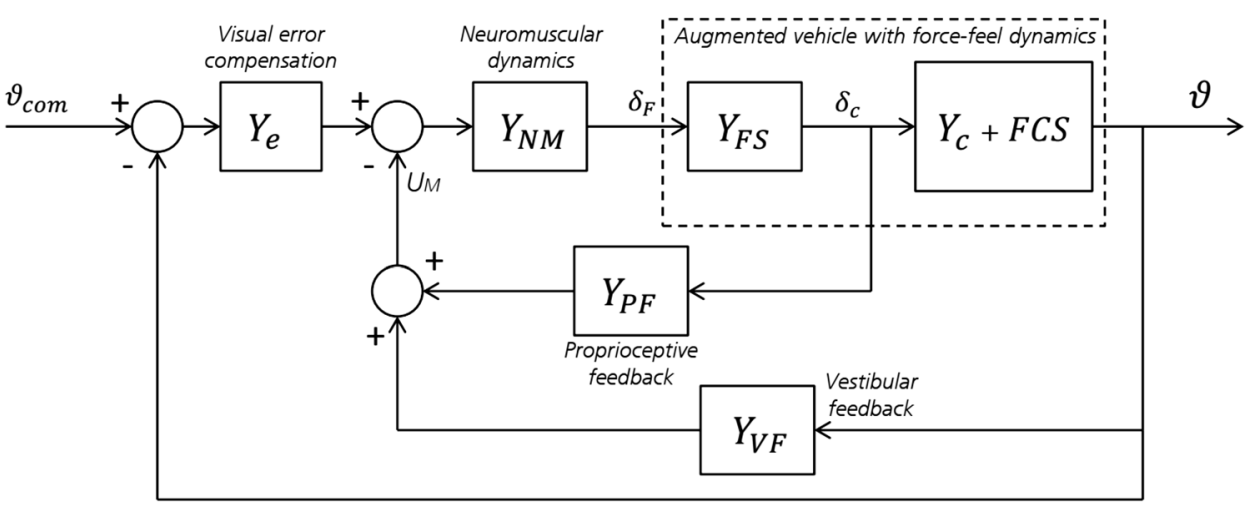


$Y_{\mathrm{FS}}=\frac{2(18.8)^{2}}{s^{2}+2(1.0) 18.8 s+18.8^{2}}$

\subsection{Vehicle dynamics approximation}

A model of DLR's Active Control Technology/Flying Helicopter Simulator (ACT/FHS) was used for real-time piloted simulation in the Air Vehicle Simulator (AVES) facility [24]. A fully non-linear model of the aircraft was used for investigations, described in Ref. [25]. For predictions using the Hess structural pilot model, low-order transfer function approximations were used. These models were identified from data collected from AVES using system identification techniques. They were used to represent the aircraft plant $Y_{\mathrm{c}}$. Three plant models were used in the investigations, to represent longitudinal and lateral dynamics during hover and forward flight (60 kts, lateral only). These are shown in Eqs. (6)-(8).

$$
\begin{aligned}
& Y_{\left.\mathrm{c}_{(\mathrm{lon} 0}\right)}=\frac{q}{\delta_{\mathrm{act}_{\mathrm{LON}}}}=\frac{1.4215}{s^{2}+(15.35) s+33.83} \\
& Y_{\left.\mathrm{c}_{\text {(ata }}\right)}=\frac{p}{\delta_{\text {act }_{L_{\mathrm{LAT}}}}}=\frac{3.0941}{s^{2}+(12.12) s+100} \\
& Y_{\left.\mathrm{c}_{\text {(ata } 0)}\right)}=\frac{p}{\delta_{\mathrm{act}_{\mathrm{LAT}}}}=\frac{3.0877}{s^{2}+(11.85) s+93.12} .
\end{aligned}
$$

In addition, the FCS was modelled and used in conjunction with the Hess pilot model. For the investigation, three different control feedback models were used: one control system with Rate Command (RC) response type and two control systems with Attitude Command response type: one deemed to have good HQs (AC(G)) and one deemed to have poor HQs $(\mathrm{AC}(\mathrm{P}))$.
Figure $6 \mathrm{a}, \mathrm{b}$ shows the longitudinal and lateral bandwidth, respectively, of the three control systems tested. For the longitudinal axis, all three models have bandwidth within the Level 1 region. For the lateral axis, all cases are within the Level 2 region, due to phase requirements of the target tracking and acquisition boundaries. The $\mathrm{AC}(\mathrm{P})$ configuration shows an apparent reduction in bandwidth from the other cases. Generally, all models were expected to exhibit sufficient bandwidth to perform MTEs. Predictions for forward flight were found to be very similar, due to similarities in the low-order equivalent transfer functions. No significant handling deficiencies were predicted using bandwidth criteria.

\subsection{Handling quality sensitivity function (HQSF)}

A further advantage of the Hess model is that the predicted HQs can be determined through the use of the HQ sensitivity function (HQSF). This is found using Eq. (9) and may be used to supplement ADS-33 predictive criteria. The HQSF is evaluated between 1 and $10 \mathrm{rad} / \mathrm{s}$, and is assessed against boundaries determined from previous investigations. The model has been employed in previous studies involving rotorcraft [20, 21]. The HQSF provides an assessment of the closed-loop pilot-vehicle model characteristics.

$H Q S F=\left|\frac{U_{\mathrm{M}}}{\theta_{\text {com }}}(j \omega) \frac{1}{K_{\mathrm{e}}}\right|$.

Figure 7a, b shows the HQSF for the three models evaluated for the hover condition. For the longitudinal dynamics the HQSF shows a clear difference between the cases. Results from the application of ADS-33E bandwidth boundaries show both the RC and $\mathrm{AC}(\mathrm{G})$ have predicted Level $1 \mathrm{HQs}$. However, the $\mathrm{AC}(\mathrm{P})$ configuration is predicted to have Level $3 \mathrm{HQs}$, due to the HQSF between 2 and $3 \mathrm{rad} / \mathrm{s}$. For the lateral dynamics, all models are predicted to have Level 1 HQs using the HQSF. A considerable difference was found
Fig. 6 Bandwidth for vehicle configurations plotted against ADS-33E target acquisition and tracking boundaries

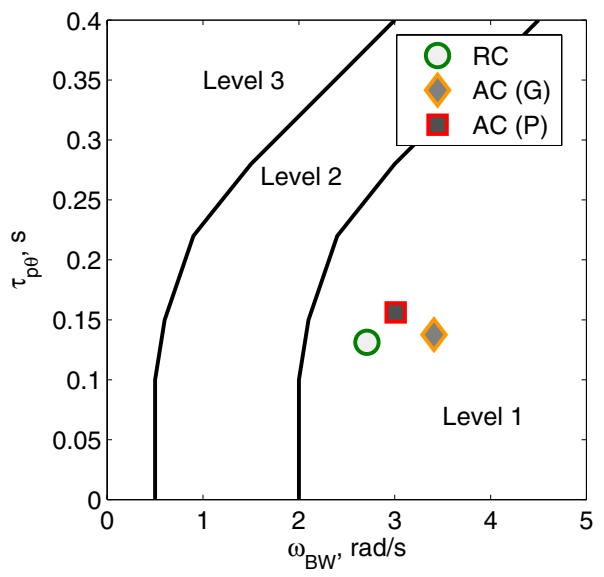

(a) Longitudinal hover case.

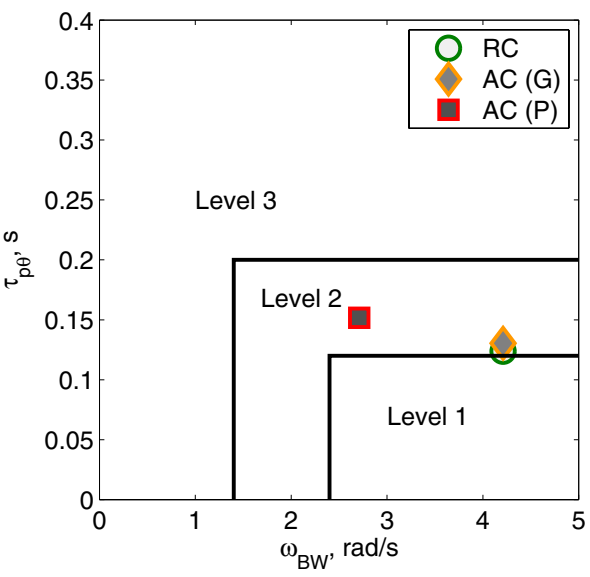

(b) Lateral hover case. 
Fig. $7 \mathrm{HQSF}$

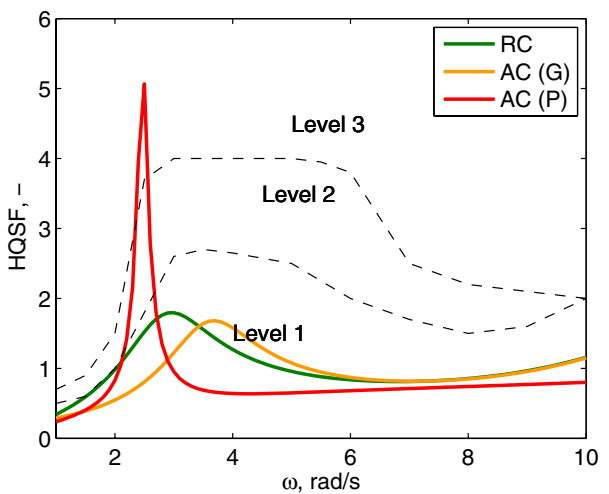

(a) HQSF, longitudinal, hover.

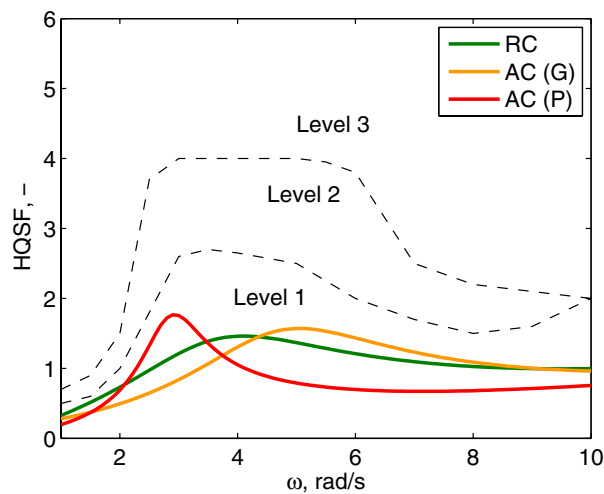

(b) HQSF, lateral, hover.

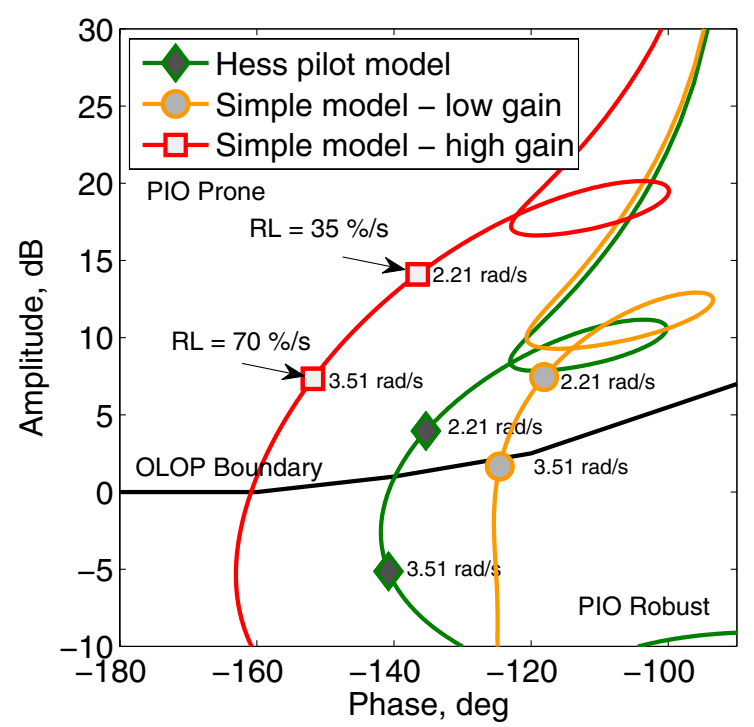

Fig. 8 OLOP predictions with three pilot models

between HQSF results (shown in Fig. 7) and bandwidth results (shown in Fig. 6). This is because bandwidth results account only for the characteristics of the open-loop vehicle. The HQSF, however, accounts for the closed-loop pilotvehicle system.

\subsection{Comparison between pure gain model and Hess pilot model}

A comparison of OLOP results, using both the Hess pilot model and the pure gain pilot model approach (both for low-gain and high-gain pilot) is shown in Fig. 8. Using the same method as outlined by Duda [11], the low- and highgain pilot models were tuned to give an open-loop phase at $0 \mathrm{~dB}$ equal to $120^{\circ}$ and $160^{\circ}$, respectively. Using the process described in the previous section, the Hess pilot model has been tuned to ensure that open-loop crossover $(0 \mathrm{~dB})$ occurs at $2 \mathrm{rad} / \mathrm{s}$. The difference in method to tune the pilot-vehicle system response leads to the significant difference between the predictions obtained. The examples show two rate limits: $35 \% / \mathrm{s}$ and $70 \% / \mathrm{s}$.

The onset points are found to be $2.21 \mathrm{rad} / \mathrm{s}$ and 3.51 $\mathrm{rad} / \mathrm{s}$, two frequencies which could realistically be reached during piloted closed-loop control. From the three pilot models shown, and using the boundary proposed by Duda [11], PIO is predicted for all $35 \% / \mathrm{s}$ rate limits. The use of the Hess model significantly changes the OLOP prediction for the $70 \% / \mathrm{s}$ case. With the Hess model, the case is clearly PIO robust. For the low gain pilot model, the case is marginally PIO robust, and for the high-gain model the model is clearly with the PIO prone region.

\section{Pilot-in-the-loop investigations}

This section details piloted simulation campaign conducted during this research effort.

\subsection{Air vehicle simulator (AVES)}

The AVES simulation facility (see Fig. 9) was used to collect all data used in this research. AVES is maintained and developed by DLR. Its design centres around the ability to easily interchange aircraft cockpits for use on a single motion platform. Currently, the facility features one fixed-wing cockpit (A320) and one helicopter cockpit (ACT/FHS). During the test campaigns conducted in this research, AVES was used without the use of the hexapod motion platform.

The AVES ACT/FHS cockpit is a replica of the aircraft. The cockpit contains four seats: one experimental pilot seat, one safety pilot seat, a flight test engineer, and a simulator operator. All experimental software to be tested in-flight is first tested in AVES. AVES is used both to support flight testing and to supplement or replace it when experimentation cannot be conducted in-flight. 


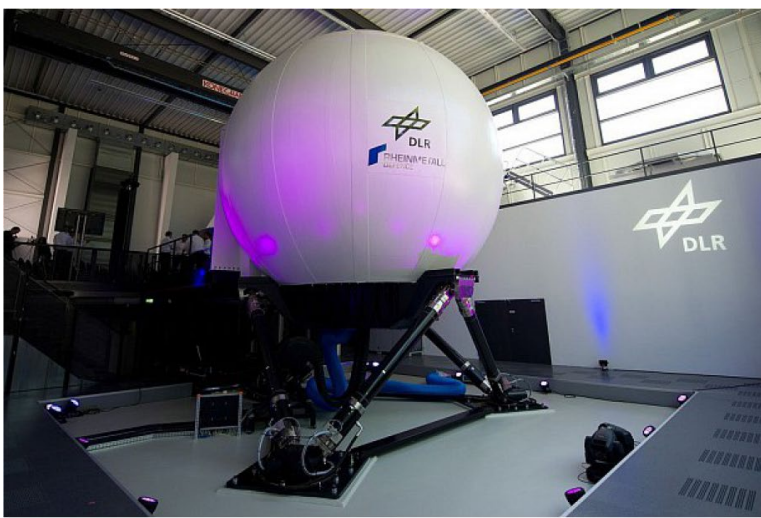

(a) AVES external view.

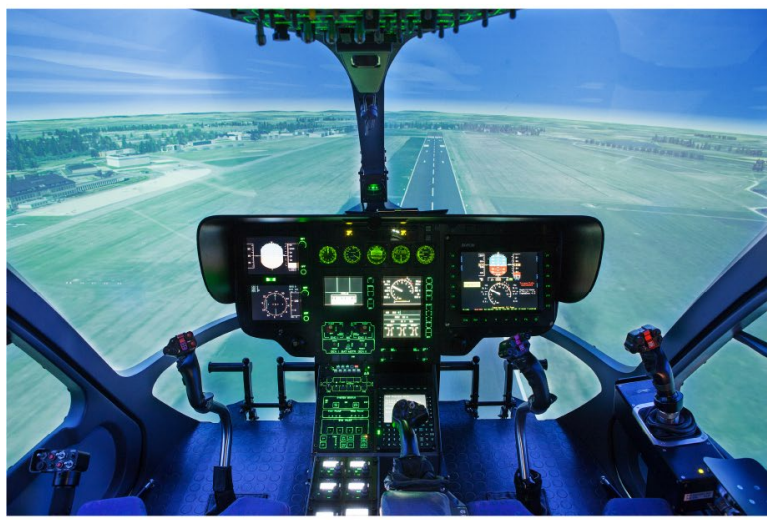

(b) AVES ACT/FHS replica cockpit.

Fig. 9 DLR's air vehicle simulator (AVES)

\subsection{Task selection}

Five tasks were selected to investigate the application of OLOP and the extensions to the model discussed above. Tasks were selected to represent different control axes and flight conditions. Information regarding the source of the tasks, flight conditions, primary axis, and modifications to the task, are shown in Table 1.

The majority of tasks were taken directly from HQ specification ADS-33E [9]. These tasks use strictly defined performance requirements, generally accepted to represent typical performance requirements for rotorcraft. As the tasks are primarily used to assess HQs, they are not directly suited to expose PIOs. Furthermore, task performance requirements were not found to be directly suited to the aircraft model used in this investigation, the ACT/FHS helicopter. Therefore, for use in this investigation, some modifications were made.

No modifications were made to the Accel-Decel or Pirouette tasks. The lateral reposition performance requirements to reach a maximum ground speed of $35 \mathrm{kts}$ was not achievable without extremely high (and unrealistic) aggression. This meant adequate performance was not achievable and pilots were required to abandon the task. Therefore, a reduction in aggression was made by reducing the required ground speed to $25 \mathrm{kts}$. This led to more suitable performance requirements. The Hover task as defined in ADS33 was found to be a low aggression task, which did not expose PIO characteristics. In Ref. [27], task aggression of the Hover manoeuvre was increased through modifications to the required hover positional tolerances. In the current investigation, this was not possible due to constraints of the visual scene. Therefore, to increase the task aggression, the translation speed was increased. This increased the aggression during the stabilisation phase, exposing PIO tendencies. Pilots stated that, although task aggression increased, it was still realistic for operations.

In addition to ADS-33 tasks, one lateral tracking task was selected. This was to investigate PIO susceptibility during forward flight. Previous PIO investigations have also utilised tracking tasks which combine changes in flight attitude and stabilisation periods $[4,14,28]$. The tracking task was completed using head-down display only, shown in Fig. 10. Using the artificial horizon, a desired attitude was displayed to the pilot. Pilots were required to capture the desired attitude within $2 \mathrm{~s}$ to achieve the task. This was set as a requirement to force pilot aggression. Once the attitude was captured, pilots were required to keep within desired (or adequate) attitude tolerances until the next change in desired attitude. The task was completed with a forward flight speed of 60 knots. The aircraft was trimmed in this condition prior to each run. The pilot was required to maintain forward

Table 1 Tasks completed in investigation

\begin{tabular}{|c|c|c|c|c|c|}
\hline Manoeuvre & Source & Flight condition & Primary axis & Modifications & Max. input (approx.) ${ }^{\mathrm{a}}, \%$ \\
\hline Accel-Decel & ADS-33E [9] & Low speed & Long. & None & $\pm 15-30$ Long. \\
\hline Lateral reposition & ADS-33E & Low speed & Lat. & $\begin{array}{l}\text { Reduction in required maximum } \\
\text { ground speed }\end{array}$ & $\pm 15-30$ Lat. \\
\hline Pirouette & ADS-33E & Low speed & Lat./long. & None & \pm 10 Lat. $/ \pm 10$ Long. \\
\hline Hover & ADS-33E & Low speed & Lat./long. & Increase in translation speed & $\pm 15-20$ Lat./5-10 Long. \\
\hline Tracking & DLR [26] & Forward flight & Lat. & None & $\pm 25-35$ Lat. \\
\hline
\end{tabular}

${ }^{\mathrm{a} O s c i l l a t o r y}$ input 


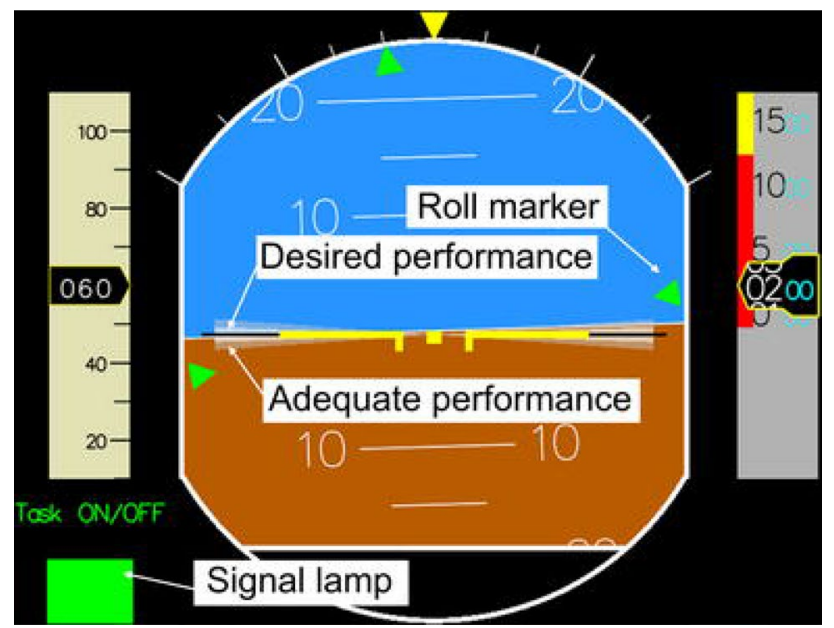

Fig. 10 Head-down display used for the tracking task

speed throughout the run. To maintain realism, no axes were fixed. Each run consisted of 14 attitude changes. Desired vehicle attitudes were between $5^{\circ}$ and $10^{\circ}$. For desired performance, pilots were required to maintain a roll attitude \pm $5^{\circ}$ and for adequate performance $\pm 10^{\circ}$. The pilots generally commented that the task was suitable and representative of aggressive forward flight manoeuvring.

To determine expected control input magnitudes, as required for the extensions to the OLOP method discussed above, a number of preliminary tests were completed with the MTEs selected. From these tests, approximate maximum control inputs were determined. A number of preliminary tests were completed for each MTE. From these tests, recorded data were visually analysed, to determine the maximum commanded input used by the pilot during completion of each task. These are shown in Table 1. For some tasks, considerable pilot input was required in both the lateral and longitudinal axes. Both input magnitudes are shown. For other tasks, only the primary axis is shown. For all OLOP predictions shown in the following section, the maximum commanded input from each task (i.e. from Table 1) was used.

It can be seen that the Accel-Decel manoeuvre required the largest inputs. These input magnitudes were found during the stabilisation element of the task following the deceleration to hover. The tracking task required the largest inputs in the lateral axis.

\subsection{Assessment methods}

To collect qualitative feedback, pilot comments were supported through the use of a number of subjective rating scales. To determine PIO incipience, two rating scales were used: the PIO rating scale and the Adverse Pilot Coupling (APC) scale. These scales are both shown in "Appendix".
The original version of the PIO scale, presented in Ref. [29] and first used in 1967 (featuring only the use of descriptive terms) was used. In this work, ratings obtained using this scale are called 'PIOR'. The 'combined scale' [30], which was developed in 1981 and fits original terms to a decision tree structure is perhaps the most frequently applied scale.

The PIOR scale is known to have a number of deficiencies, which lead to difficulties when classifying PIOs. These are discussed in detail in Refs. [31, 32]. These include the lack of guidance for pilots, lack of consideration for nonoscillatory phenomena (e.g. rapid divergence, loss of control) and the absence of terms regarding the severity of oscillations. In Ref. [27], the APC scale was presented as an alternative means of assessing the broader field of Rotorcraft-Pilot Couplings (RPCs). This was developed through investigations during the ARISTOTEL project [4]. The APC scale was developed with experimental test pilots, and detailed investigations comparing results obtained using previous scales were conducted [27]. In addition, HQ ratings were obtained using the Cooper-Harper scale.

\section{Results}

This section shows the results obtained from completion of the selected tasks.

\subsection{Accel-Decel}

The Accel-Decel manoeuvre was completed using only the RC control system. During completion of the manoeuvre, both pilots commented that the task aggression was too high (to safely complete the manoeuvre in-flight). To achieve desired ground speed, as specified by the task performance requirements, very large nose-down pitch attitudes were required. This was considered unrealistic for operations close to the ground. If the investigation were repeated, the longitudinal course track should be lengthened, to allow for lower aggression. Table 2 shows the subjective ratings obtained during completion of the Accel-Decel.

During completion of the Accel-Decel manoeuvre, both pilots did not encounter large and clearly visible PIOs. With the additional rate limiting, pilot ratings suggested mild oscillations (or motions) in the longitudinal axis occurred during the stabilisation period of the task. These also appeared to influence task performance, and hindered the pilots' ability to obtain desired performance standards. However, oscillations did not lead to uncontrollable vehicle characteristics. Figure 11 shows an example of data recorded during the completion of the Accel-Decel manoeuvre. Here, two examples of cases flown with RLEs: $35 \% / \mathrm{s}$ and $17 \% / \mathrm{s}$ in the longitudinal channel. 
Table 2 Pilot rating: AccelDecel task

\begin{tabular}{lllllll}
\hline Pilot & Com & RL & $\omega_{\text {OLOP }}$ & HQR & APCR & PIOR \\
\hline A & RC & N & - & 4 & 2 & 2 \\
A & RC & 35 & 2.4 & 5 & $4 \mathrm{~A}$ & 2 \\
A & RC & 17 & 1.4 & 5 & $4 \mathrm{~A}$ & 2 \\
B & RC & N & - & 4 & 1 & 1 \\
B & RC & 35 & 2.4 & 5 & 4 C & 2 \\
B & RC & 17 & 1.4 & 5 & 4 B & 2 \\
\hline
\end{tabular}
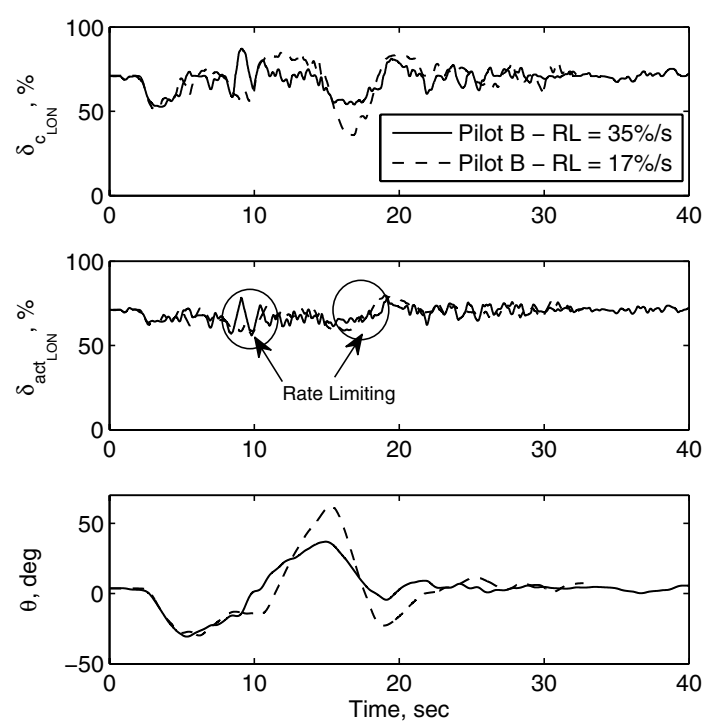

Fig. 11 Data from completion of Accel-Decel task

It can be seen that RLE were 'activated' during completion of the task. However, divergent and large PIOs were not experienced. APC ratings obtained suggest that small minor oscillations were apparent, but did not lead to any significant change in control strategy. A degradation in HQR was also found, from a HQR 4 to HQR 5 when introducing RLEs.

Figure 12 shows a comparison between the classical and task-specific (TS) OLOP predictions for the Accel-Decel task. Points shown are the $\omega_{\mathrm{OLOP}}$ for the two RL values tested: $35 \% / \mathrm{s}$ and $17 \% / \mathrm{s}$. For this case, all points are predicted to be PIO prone. Also shown are APC ratings awarded during the task completion.

As shown, for both the classical and TS OLOP, $\omega_{\mathrm{OLOP}}$ points are above the OLOP boundary. This means the case is predicted to be Cat. II PIO prone. Using the classical method, the $\omega_{\text {OLOP }}$ points are significantly above the boundary. However, clear PIOs were not experienced during completion of the Accel-Decel manoeuvre. Using the TS method, $\omega_{\mathrm{OLOP}}$ points are close to the OLOP boundary. This appears to be more representative of results found during piloted simulation.

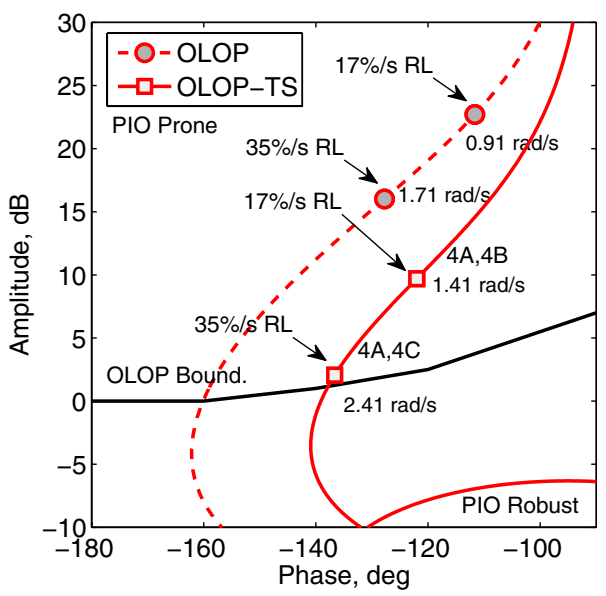

Fig. 12 OLOP predictions for Accel-Decel task

\subsection{Tracking task}

The tracking task was conducted using all three control command types and flown by both pilots. Ratings awarded are shown in Table 3. During completion of the tracking task, the vehicle was trimmed in forward flight. Performance requirements were only in the lateral axis. The capture element of the task required the pilots to command large control input displacement. This was found to successfully expose PIOs during the tests, however, not consistently for both pilots. During completion of the task, Pilot B was more resistant to PIO, particularly for the control types with predicted good HQs (RC and $\mathrm{AC}(\mathrm{G})$ ).

Figure $13 \mathrm{a}, \mathrm{b}$ shows two examples of the results obtained from completion of the tracking task for Pilot A and Pilot B, respectively. These results were obtained for the $\mathrm{AC}(\mathrm{G})$ control system. Figure $13 \mathrm{a}$ shows the results where Pilot A entered large PIOs and clearly activated vehicle RLE. Roll oscillations are shown in the vehicle roll attitude output $(\phi)$ after approximately $t=35 \mathrm{~s}$. These continue until the completion of the manoeuvre. In this case, the pilot is unable to arrest oscillations during attempted completion of the task. These oscillations can clearly be classified as Cat. II, as rate limiting is apparent throughout the run. Conversely, Fig. 13b shows results from the same case when performed by Pilot B. In this case, considerable 
Table 3 Pilot rating: tracking task

\begin{tabular}{lllllll}
\hline Pilot & Com & RL & $\omega_{\text {OLOP }}$ & HQR & APCR & PIOR \\
\hline A & RC & 35 & 2.4 & 3 & 1 & 1 \\
A & RC & 17 & 1.2 & 3 & 1 & 1 \\
A & RC & 5 & 0.4 & 5 & $5 B$ & 2 \\
B & RC & 17 & 1.2 & 5 & 2 & 2 \\
A & AC(G) & N & - & 4 & 1 & 2 \\
A & AC(G) & 35 & 1.3 & 5 & 1 & 1 \\
A & AC(G) & 17 & 0.8 & 6 & $7 \mathrm{~B}$ & 4 \\
B & AC(G) & N & - & 5 & $4 \mathrm{~A}$ & 2 \\
B & AC(G) & 17 & 0.8 & 4 & 2 & 2 \\
B & AC(G) & 10 & 0.6 & 5 & $4 \mathrm{~A}$ & 2 \\
A & AC(P) & N & - & 5 & $5 B$ & 3 \\
A & AC(P) & 35 & 1.1 & 6 & $7 \mathrm{~B}$ & 4 \\
A & AC(P) & 17 & 0.8 & 7 & $7 \mathrm{~B}$ & 4 \\
B & AC(P) & N & - & 4 & $4 \mathrm{~A}$ & 2 \\
B & AC(P) & 17 & 0.8 & 6 & $5 \mathrm{~A}$ & 2 \\
\hline
\end{tabular}

rate limiting also occurs throughout the completion of the task. However, the pilot consciously uses a control strategy to suppress (or avoid) PIO. In this case, the pilot reduces his control gain and applies no immediate large change in control input following any large change in vehicle roll attitude. This affectively means that the pilot is going 'open-loop' and, therefore, avoiding PIO. Despite this strategy, the pilot is able to complete the task to desired performance requirements. At the end of the manoeuvre, during a large attitude capture, a small oscillation in roll attitude is apparent. The pilot did not recognise this as PIO during the manoeuvre. Results here, and the difference between the two pilots, suggest that the manoeuvre should be further constrained to consistently expose underlying PIOs.

In the case discussed above, it was possible for the pilot to avoid PIO due to the favourable HQs of the vehicle. However, for the PVS with poor HQs (i.e. AC(P)), this was not possible. Figure 14 shows the completion of the manoeuvre performed by Pilot $\mathrm{B}$ with the $\mathrm{AC}(\mathrm{P})$ model. As shown, following $t=60 \mathrm{~s}$, rate limiting severity and frequency increases. Furthermore, roll oscillations are shown in the roll channel. For the same configuration, Pilot A entered severe PIOs, which forced him to abandon the task.

Figure 15a shows the TS OLOP predictions for the tracking task. Predictions are for the lateral axis, calculated using the forward-flight model. OLOP predictions are shown for cases with $\mathrm{RL}=35 \% / \mathrm{s}$ and $\mathrm{RL}=17 \% / \mathrm{s}$ for all control system models. Also shown are APC ratings awarded by both pilots. Results show disagreement between pilot ratings and the OLOP boundary. This is found for cases with large phase margin at the open-loop gain crossover (Amplitude
$0 \mathrm{~dB}$ ). These cases were found to be PIO robust during the completion of the tracking task. The case where the phase margin was small $(\mathrm{AC}(\mathrm{P}))$ was found to result in severe PIOs $(\mathrm{APCR}=7 \mathrm{~B}, \mathrm{PIOR}=5)$.

\subsection{Lateral reposition}

The lateral reposition task was completed by one pilot (Pilot A). This was due to available time during the test campaign. The pilot completed the manoeuvre using two control types (RC and $\mathrm{AC}(\mathrm{P})$ ). Ratings awarded are shown in Table 4.

For the RC system, no significant PIOs were observed. Though the APCRs, the pilot commented that he experienced non-oscillatory motions during the completion of the task. Conversely, severe PIOs were experienced using the $\mathrm{AC}(\mathrm{P})$ model. In this case, reduction in RL led to a significant increase in PIO severity. For the case with $\mathrm{RL}=17 \% / \mathrm{s}$, the pilot awarded APCR $=8 \mathrm{E}$ and PIOR $=5$. In this case, the pilot abandoned the task due to oscillations experienced. These occurred during the attempted stabilisation following the lateral translation. Abandoning the task led to the convergence of oscillations and stabilisation of the vehicle.

OLOP predictions for the lateral reposition task are shown in Fig. 15b. Predictions are similar to those found for the tracking task. In this case, the OLOP boundary appears to be conservative for cases with high-phase margin at gain crossover.

\subsection{Pirouette}

The Pirouette manoeuvre was completed by both test pilots, using all control configurations. In this task, pilots were 

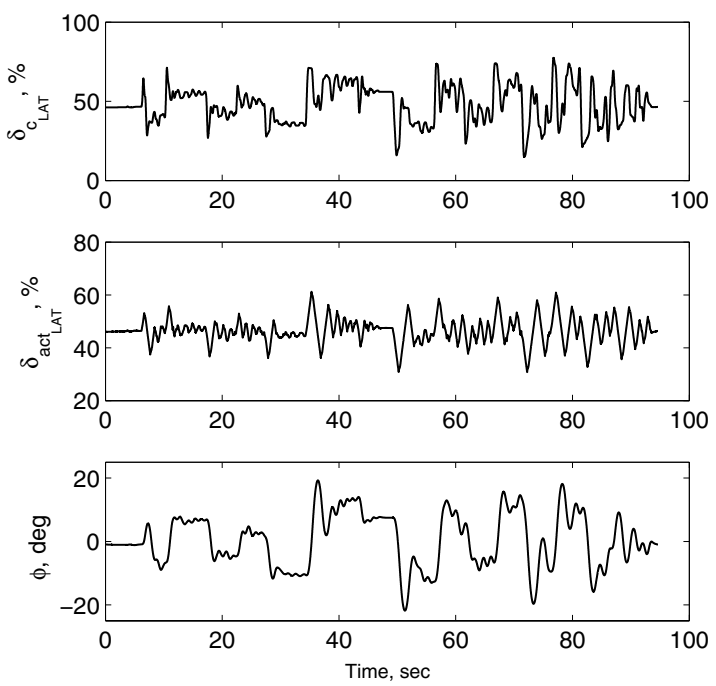

(a) Pilot $\mathrm{A}, \mathrm{AC}(\mathrm{G}), \mathrm{RL}=17 \% / \mathrm{s}, \mathrm{HQR}=6, \mathrm{APC}=7 \mathrm{~B}$, $\mathrm{PIOR}=4$.
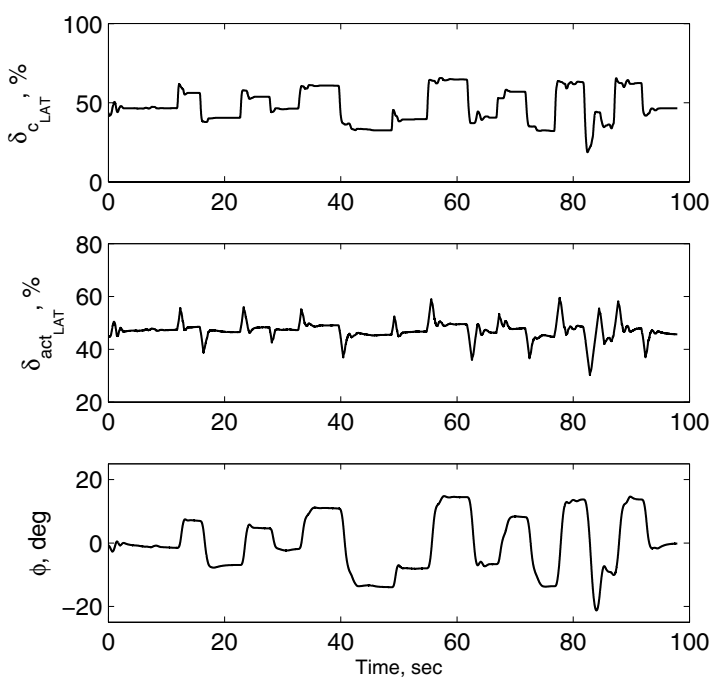

(b) Pilot $\mathrm{B}, \mathrm{AC}(\mathrm{G}), \mathrm{RL}=17 \% / \mathrm{s}, \mathrm{HQR}=4, \mathrm{APC}=2$, $\mathrm{PIOR}=2$

Fig. 13 Examples of data obtained during the tracking task

required to apply control inputs both in lateral and longitudinal channels. Primary and largest control inputs were made within the lateral axis, particularly during the stabilisation element of the task. Pilots were constantly required to correct the longitudinal position whilst completing the manoeuvre. Results from pilot subjective assessment are shown in Table 5.

Unexpectedly, the task requirements of the Pirouette manoeuvre consistently caused longitudinal PIOs. These were found as the pilots attempted to maintain their
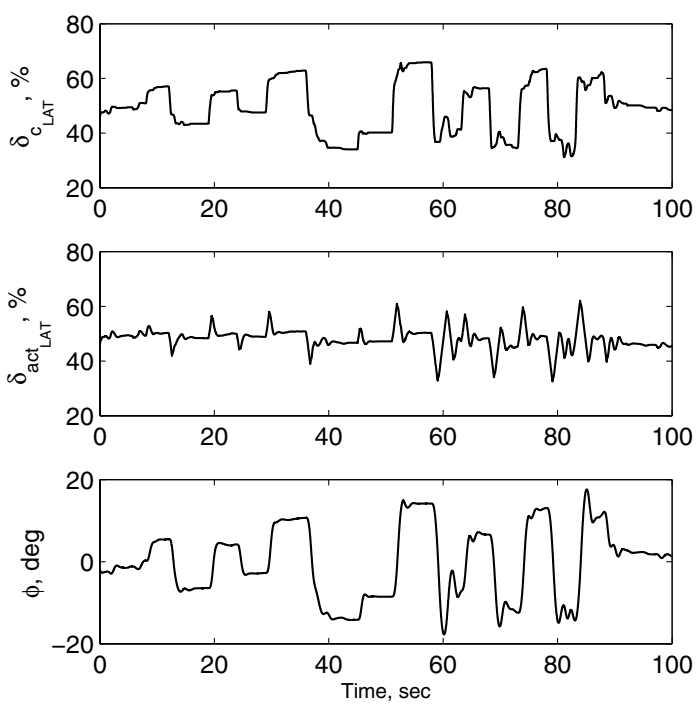

Fig. 14 Example of PIO: Pilot $\mathrm{B}, \mathrm{AC}(\mathrm{P}), \mathrm{RL}=17 \% / \mathrm{s}, \mathrm{HQR}=6$, $\mathrm{APC}=5 \mathrm{~A}, \mathrm{PIOR}=2$

longitudinal track position whilst maintaining the lateral translation. Figure $15 \mathrm{c}$ shows OLOP predictions for the longitudinal axis.

For the $\mathrm{AC}(\mathrm{P})$ configuration, only a very small phase margin exists at the $0 \mathrm{db}$ crossover point (difference between $-180^{\circ}$ and phase at $0 \mathrm{~dB}$, Fig. $\left.15 \mathrm{c}\right)$. This indicates that the PVS is close to instability. It is believed that the proximity to instability and the task performance requirements have led to activation of RLE. An example of a divergent oscillation which occurred during completion of the task is shown in Fig. 16a.

Although the majority of PIOs during the Pirouette manoeuvre were found in the longitudinal axis, one PIO was observed in the lateral axis. This was found during a test completed by Pilot A, using the $\mathrm{AC}(\mathrm{P})$ command system. With the $\mathrm{RL}=17 \% / \mathrm{s}$, the pilot entered a small, rate-limited PIO during the hover capture, and the end of the manoeuvre. This is shown in Fig. 16b. The pilot stated that, for this case, the PIO was not severe, and awarded APC 4C. As shown, in this case, the PIO started following small oscillatory control inputs at $t=43 \mathrm{~s}$. During the start of these oscillations, the pilot commands control inputs approximately $\pm 3-4 \%$ of available lateral control.

Figure 15d shows the OLOP prediction for this case: lateral dynamics of the vehicle in hover, with pilot input magnitude of $\pm 4 \%$. As shown, using the classical OLOP boundary, the case is predicted to be marginally PIO Prone, confirming the results of piloted assessment. 
Fig. 15 OLOP predictions using task-specific (TS) approach

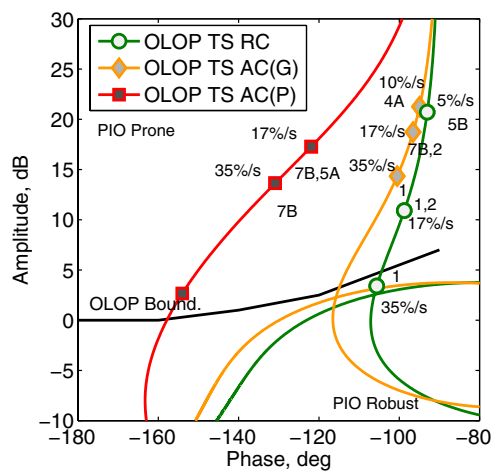

(a) OLOP predictions: Tracking task.

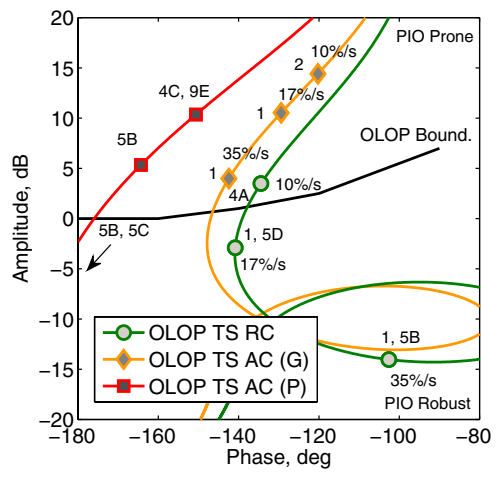

(c) OLOP predictions: Pirouette - longitudinal.

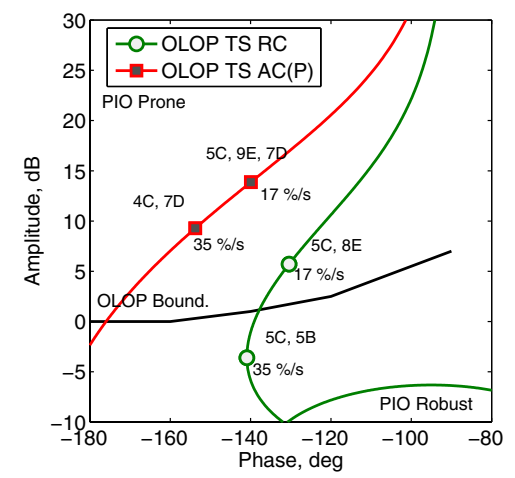

(e) OLOP predictions: Hover - longitudinal.

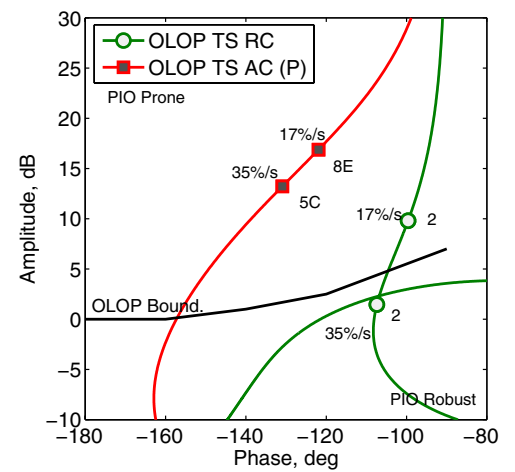

(b) OLOP predictions: Lateral Reposition.

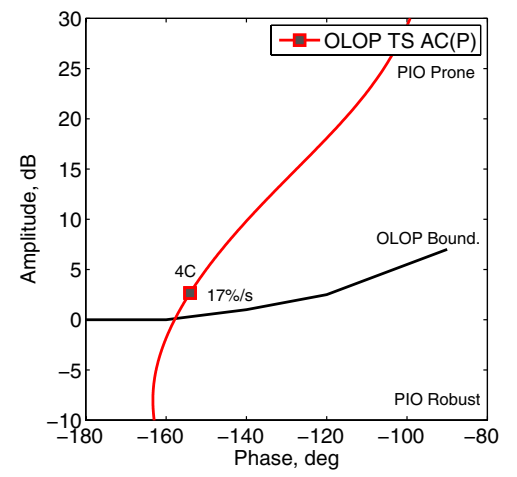

(d) OLOP predictions: Pirouette - lateral.

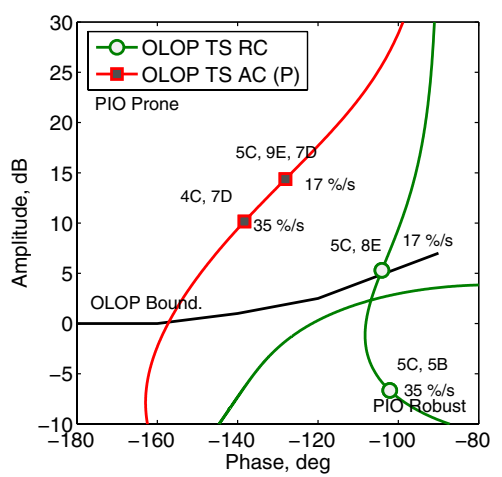

(f) OLOP predictions: Hover - lateral.
Table 4 Pilot rating: lateral reposition

\begin{tabular}{lllllll}
\hline Pilot & Com & RL & $\omega_{\text {OLOP }}$ & HQR & APCR & PIOR \\
\hline A & RC & N & - & 4 & 2 & 2 \\
A & RC & 35 & 2.7 & 5 & 2 & 2 \\
A & RC & 17 & 1.3 & 5 & 2 & 2 \\
A & AC(P) & 35 & 1.1 & 6 & $5 \mathrm{C}$ & 3 \\
A & AC(P) & 17 & 0.8 & 7 & $8 \mathrm{E}$ & 5 \\
\hline
\end{tabular}


Table 5 Pilot rating: pirouette

\begin{tabular}{lllllll}
\hline Pilot & Com & RL & $\omega_{\text {OLOP lat/long }}$ & HQR & APCR & PIOR \\
\hline A & RC & 35 & $7.7 / 5.0$ & 5 & $5 B$ (lon) & 4 (lon) \\
A & RC & 17 & $4.0 / 3.1$ & 7 & 5 D (lon) & 6 (lon) \\
B & RC & N & $-/-$ & 4 & 1 & 1 \\
B & RC & 35 & $7.7 / 5.0$ & 4 & 1 & 1 \\
B & RC & 17 & $4.0 / 3.1$ & 4 & 1 & 1 \\
B & RC & 10 & $2.3 / 2.2$ & 4 & $4 A$ (lon) & 2 (lon) \\
A & AC(G) & N & $-/-$ & 5 & 1 & 1 \\
A & AC(G) & 35 & $3.6 / 2.8$ & 4 & 1 & 1 \\
A & AC(G) & 17 & $1.9 / 1.8$ & 4 & 1 & 1 \\
B & AC(G) & N & $-/-$ & 4 & 1 & 1 \\
B & AC(G) & 35 & $3.6 / 2.8$ & 4 & 1 & 1 \\
B & AC(G) & 17 & $1.9 / 1.8$ & 4 & 1 & 1 \\
B & AC(G) & 10 & $1.3 / 1.3$ & 4 & 2 & 2 \\
A & AC(P) & N & $-/-$ & 5 & $5 B$ (lon) & 4 (lon) \\
A & AC(P) & 17 & $1.4 / 1.3$ & 5 & $4 C$ (lon) & 4 (lon) \\
B & AC(P) & N & $-/-$ & 6 & $5 C$ (lon) & 3 (lon) \\
B & AC(P) & 35 & $2.1 / 1.8$ & 5 & $5 B$ (both) & 3 (both) \\
B & AC(P) & 17 & $1.4 / 1.3$ & 10 & $9 E$ (both) & 6 (both) \\
\hline
\end{tabular}

\subsection{Hover}

The Hover task was conducted using both the RC and the $\mathrm{AC}(\mathrm{P})$ control systems. Unlike the other tasks performed, PIOs were consistently experienced both in the lateral and longitudinal axes. Table 6 shows the results obtained. Additional information regarding whether RLEs were 'activated' during completion of the manoeuvre is also shown, to determine whether APC and PIO ratings awarded resulted from longitudinal or lateral characteristics.

In many cases, both the lateral and longitudinal RLEs were triggered during completion of the Hover MTE. In general, HQRs for the Hover task, with the faster transition speed, indicated that pilots had difficulty achieving desired and adequate task performance standards. The inclusion of RLEs led to a degradation in HQRs. During completion of the Hover MTE, on one occasion, PIOs led to a loss of control. This was for the $\mathrm{AC}(\mathrm{P})$ case, whereby divergent oscillations began in the longitudinal axis and subsequently caused oscillations to also occur in the lateral axis. Using the RC model, limiting in the lateral axis was more apparent. This was due to the larger control inputs required to complete task performance, particularly during the stabilisation element, in this axis.

OLOP predictions, using approximations of maximum control input expected during completion of the Hover task are shown in Fig. 15e, f, for both longitudinal and lateral dynamics, respectively. Also shown are the APCRs obtained during task completion. For each case, pilots awarded only a single APC rating. Therefore, this is repeated in each OLOP figure.
On one occasion, the Pilot awarded a APCR $=8 \mathrm{E}$, for a PIO experienced in the lateral axis during completion of the task. This PIO is shown in Fig. 17 and occurred during the attempted stabilisation element of the task. Oscillations continued for only 1.5 cycles. The pilot stated that to arrest the severe oscillations, he was required to 'abandon the task'. As a result, and following the guidelines on the use of the APC scale, he awarded APC $=8 \mathrm{E}$. However, following the initial oscillation, the pilot was able to stabilise the vehicle, and complete the stabilised hover element of the task.

For this case, the pilot could not successfully complete the translation to hover in the allowable time, due to the PIO which occurred during the attempted capture. Large oscillations led to a large change in ground speed between 6 and 10 knots during the attempted capture. For this case, severe PIOs were not expected, due to the proximity of the $\omega_{\text {OLOP }}$ to the classical boundary. For the other tasks, this region was found to be PIO robust, both in terms of longitudinal and lateral PIO dynamics. This was the only case found during the investigation where a PIO was found for a case close to the classical OLOP boundary with an open-loop phase above $-120^{\circ}$.

\subsection{Summary of results}

Generally, the extensions to the OLOP model were found to be suitable additions to the Cat. II PIO prediction method. The results obtained reflected subjective feedback from the experimental test pilots. Using the Hess pilot model, a greater understanding of both the open-loop and closedloop dynamics of the PVS was obtained. Unlike the classical 

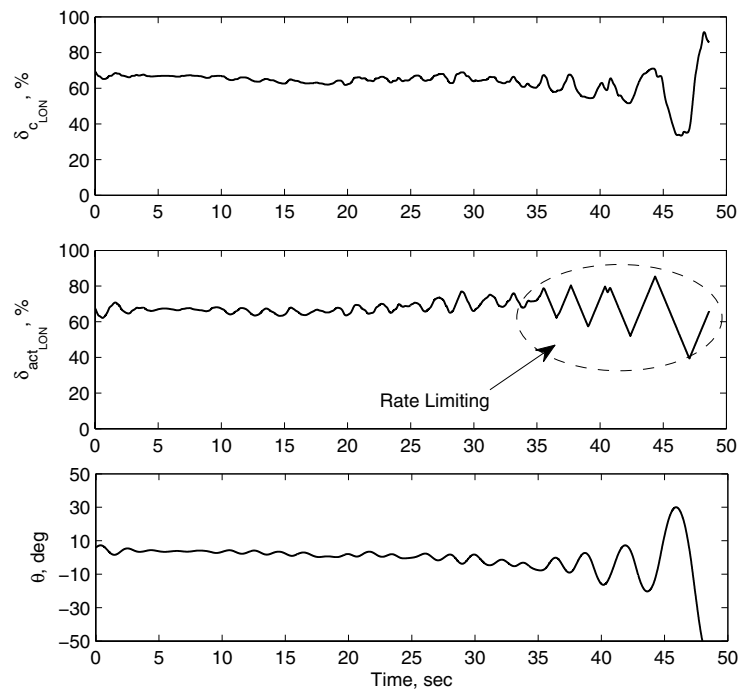

(a) Divergent longitudinal PIO during manoeuvre.
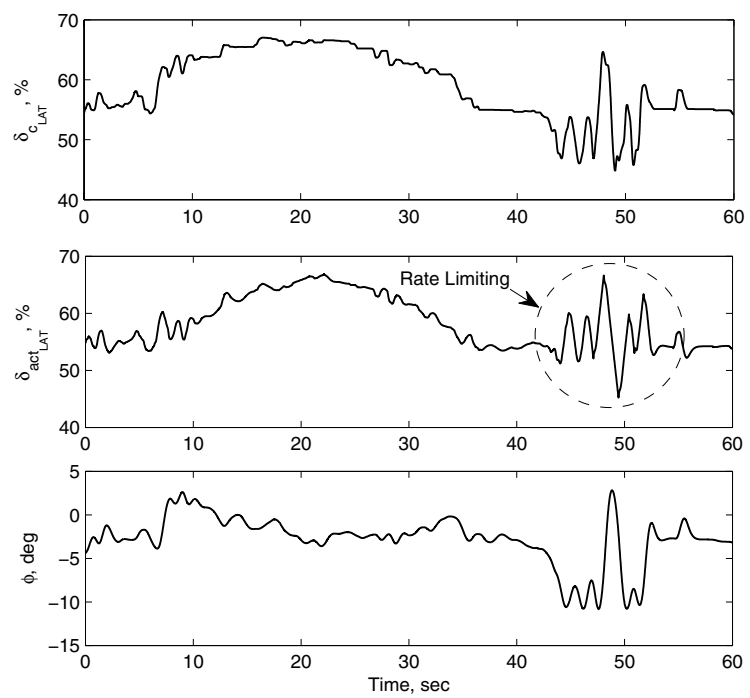

(b) Lateral PIO during completion of manoeuvre.

Fig. 16 Examples of data obtained during the Pirouette manoeuvre
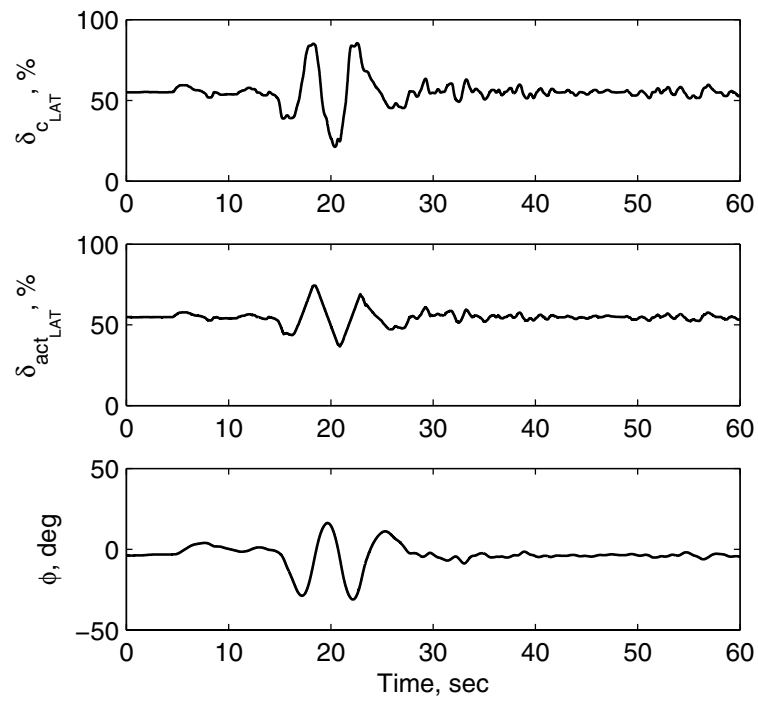

Fig. 17 Lateral PIO occurring during Hover MTE

OLOP method, the PVS dynamics were tuned using a gain crossover frequency. Vehicle models with high-open-loop phase margin were found to be more robust to PIO than those with a low-phase margin. This is in agreement with the classical OLOP boundary. Figure 18 shows $\omega_{\text {OLOP }}$ points obtained from OLOP analysis, for all tasks and both lateral and longitudinal axes. Each $\omega_{\text {OLOP }}$ point has been classified whereby either no Cat. II PIO was experienced or where Cat. II PIOs were experienced. These classifications are based upon the pilot subjective ratings awarded.

Figure 18 shows the classical OLOP boundary, proposed by Duda [11], and a proposed boundary based upon results obtained in this investigation. The results from this investigation showed that points featuring high-open-loop phase margin were found to be PIO robust when completing the majority of MTEs. One point (lateral axis, Hover task) was found to result in a severe PIO, but this was not sustained,

Table 6 Pilot rating: Hover

\begin{tabular}{llllllll}
\hline Pilot & Com & RL & $\omega_{\text {OLOP }}$ lat/long & HQR & APCR & PIOR & RL active \\
\hline A & RC & 35 & $4.1 / 3.2$ & 6 & $5 \mathrm{C}$ & 4 & No \\
A & RC & 17 & $2.0 / 1.9$ & 7 & $5 \mathrm{C}$ & 4 & Lat \\
A & RC & 17 & $2.0 / 1.9$ & 7 & $5 \mathrm{C}$ & 4 & Both \\
B & RC & N & $-1-$ & 5 & $4 \mathrm{~A}$ & 2 & No \\
B & RC & 35 & $4.1 / 3.2$ & 6 & $5 B$ & 3 & No \\
B & RC & 17 & $2.0 / 1.9$ & 7 & $8 \mathrm{E}$ & 6 & Both \\
A & AC(P) & 35 & $1.4 / 1.4$ & 4 & $4 \mathrm{C}$ & 2 & No \\
A & AC(P) & 17 & $1.0 / 1.0$ & 4 & $5 \mathrm{C}$ & 4 & Both \\
B & AC(P) & N & $-1-$ & 5 & $5 B$ & 3 & No \\
B & AC(P) & 35 & $1.4 / 1.4$ & 7 & $7 \mathrm{D}$ & 4 & Long \\
B & AC(P) & 17 & $1.0 / 1.0$ & 10 & $9 \mathrm{E}$ & 6 & Both \\
B & AC(P) & 17 & $1.0 / 1.0$ & 9 & $7 D$ & 4 & Both \\
\hline
\end{tabular}




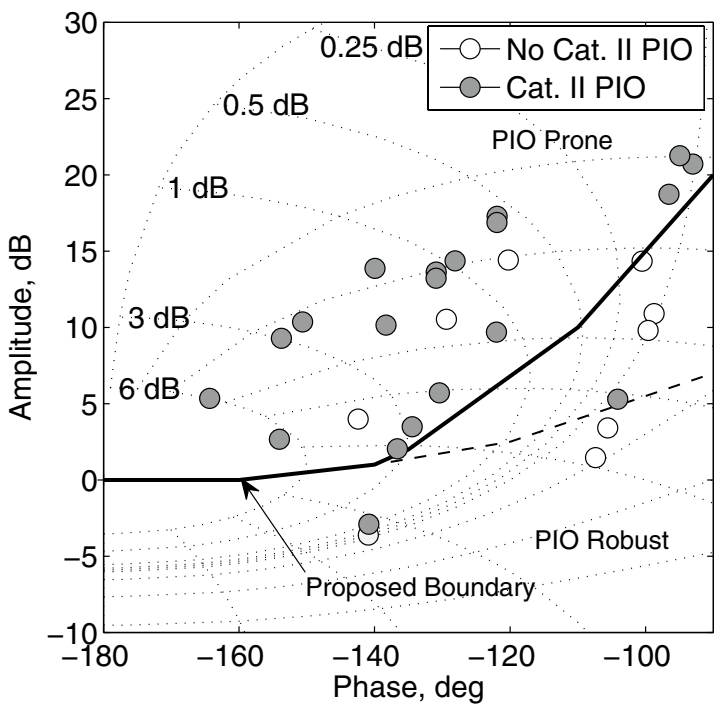

Fig. 18 Proposed boundary based on results obtained from study

continuing for only for 1.5 cycle. As a result, the findings generally supported increasing the slope of the OLOP boundary with increasing phase margin. The proposed boundary, based on the results obtained, was found to be very similar to the boundary proposed for phase-compensated RLEs in Ref. [17].

\section{Conclusions}

This study has shown that the Open-Loop Onset Point criterion (OLOP) can be successfully applied to rotorcraft to predict the incipience of Cat. II PIO events. The following are the key conclusions from this investigation.

- The five tasks investigated in this research effort were found to be suitable to expose Cat. II PIOs. A number of tasks were modified from manoeuvre specifications contained in the Handling Qualities (HQ) specification ADS-33E. These modifications improved the suitability of the tasks to expose PIOs. Although generally tasks were appropriate, a number of improvements were suggested for future investigations. Furthermore, in some cases, task constraints must be revisited, to ensure that pilot aggression is sufficient to trigger PIOs.

- Extensions to the OLOP method, both the use of a sophisticated pilot model and realistic control input magnitudes was found to lead to appropriate PIO predictions for the cases tested in this research effort. Using the OLOP boundary, results obtained were much less conservative in comparison to those found when using the 'classical OLOP' method.
- Results from the investigation were used to generate a modified OLOP boundary. During the investigation, it was found that configurations featuring a high-phase margin at the gain crossover were more robust to PIO. Therefore, the results suggested that the OLOP boundary should be adjusted at phase values greater than $-140^{\circ}$.

This study has demonstrated an update to the OLOP method through use of realistic control inputs and a sophisticated pilot model. An extension to this methodology would be to characterise the pilot in the frequency domain. The characterisation would be used to update the parameters of the pilot model, replacing the approximations used in this research effort. This is recommended for future research.

Although the method has been developed and demonstrated using a helicopter model, there is nothing limiting the further application to other types of vehicle, both rotorcraft [e.g. Tiltrotor, electric vertical take-off and lift (eVTOL)] and fixed-wing. Particularly for new aircraft, during flight testing, the method can offer significant benefit by giving clear guidance on how to tune and configure rate limiters to avoid PIOs. Research in this area is recommended for future investigations.

Acknowledgements Open Access funding provided by Projekt DEAL. Data used in this paper were collected during a joint test campaign between researchers from DLR and the National Research Council Canada (NRC). The author wishes to thank Marc Alexander (NRC) for his assistance conducting the campaign in the AVES simulator. Further thanks is extended to test pilots who participated in the campaign, Perry Comeau (NRC) and Miles Barnett (DLR). Finally, the author wishes to thank Holger Duda, for his advice regarding the application of the OLOP criterion.

Open Access This article is licensed under a Creative Commons Attribution 4.0 International License, which permits use, sharing, adaptation, distribution and reproduction in any medium or format, as long as you give appropriate credit to the original author(s) and the source, provide a link to the Creative Commons licence, and indicate if changes were made. The images or other third party material in this article are included in the article's Creative Commons licence, unless indicated otherwise in a credit line to the material. If material is not included in the article's Creative Commons licence and your intended use is not permitted by statutory regulation or exceeds the permitted use, you will need to obtain permission directly from the copyright holder. To view a copy of this licence, visit http://creativecommons.org/licenses/by/4.0/.

\section{Appendix}

The appendix contains rating scales used in this investigation: the Adverse Pilot Coupling (APC) rating scale and the Pilot-Induced Oscillation (PIO) rating scale (Figs. 19, 20). 


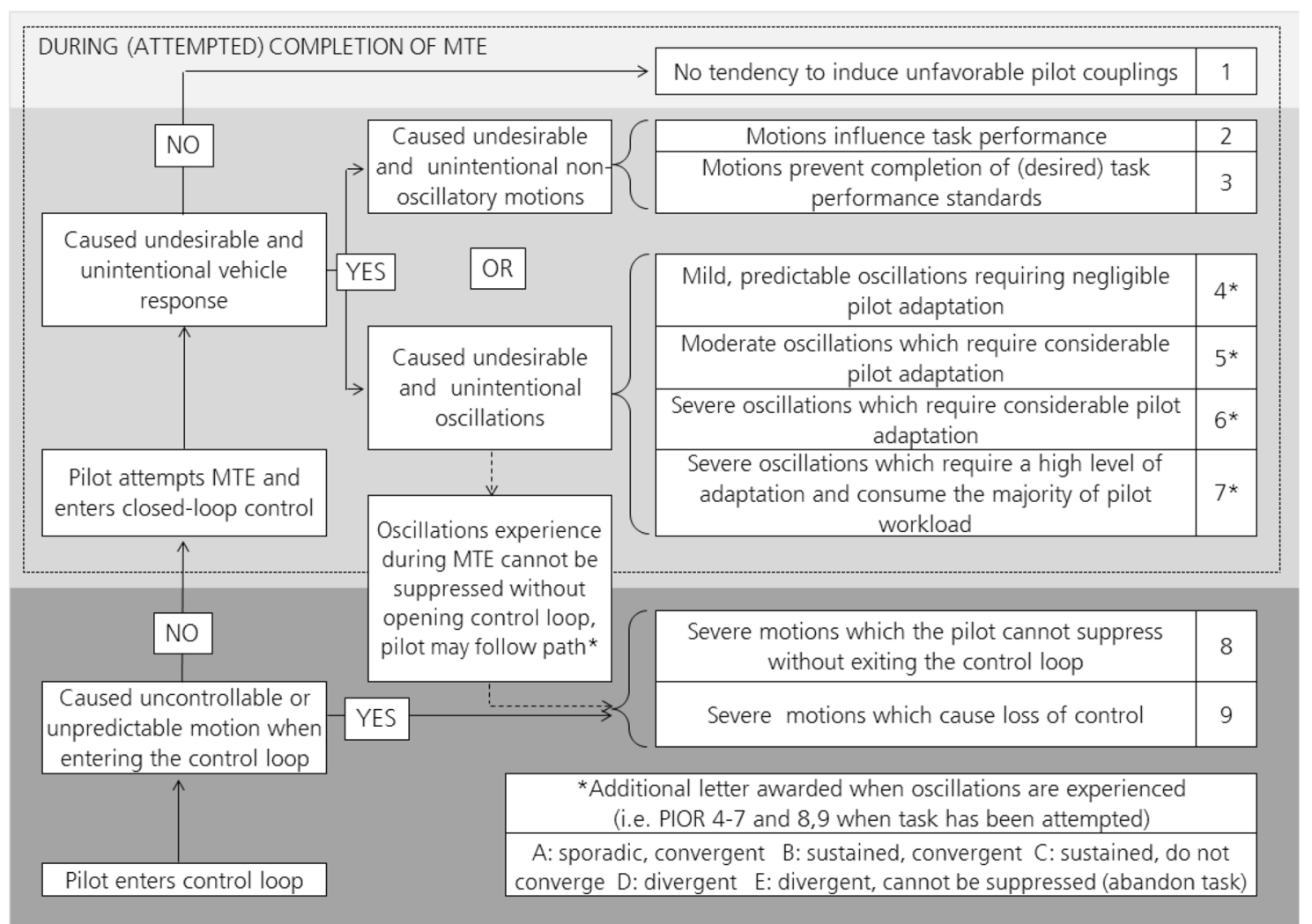

Fig. 19 Adverse pilot coupling rating scale (APCR)

Fig. 20 Pilot-induced oscillation rating scale (PIOR)

\begin{tabular}{|l|c|}
\hline Description & Rating \\
\hline No tendency for pilot in induce undesirable motions & 1 \\
\hline $\begin{array}{l}\text { Undesirable motions tend to occur when pilot initiates abrupt manoeuvres or attempts } \\
\text { tight control. These motions can be prevented or eliminated by pilot technique }\end{array}$ & 2 \\
\hline $\begin{array}{l}\text { Undesirable motions easily induced when pilot initates abrupt manoeuvres or attepts } \\
\text { tight control. These motions can be prevented or eliminated but only at the sacrifice to } \\
\text { task performance or through considerable pilot attention and effort }\end{array}$ & 3 \\
\hline $\begin{array}{l}\text { Oscillations tend to develop when pilot initiates abrupt manoeuvres or attempts tight } \\
\text { control. Pilot must reduce gain or abandon task to recover. }\end{array}$ & 4 \\
\hline $\begin{array}{l}\text { Divergent oscillations tend to develop when pilot initiates abrupt manouevres or attepts } \\
\text { tight control. Pilot must open loop by releasing or freezing the stick. }\end{array}$ & 5 \\
\hline $\begin{array}{l}\text { Disturbance or normal pilot control may cause divergent oscillations. Pilot must open } \\
\text { control loop by releasing or freezing the control stick. }\end{array}$ & 6 \\
\hline
\end{tabular}

\section{References}

1. McRuer, D.T., Droste, C.S., Hansman, R.J., Hess, R.A., LeMaster, D.P., Matthews, S., McDonnell, J.D., McWha, J., Melvin, W.W., Pew, R.W.: Aviation Safety and Pilot Control: Understanding and Preventing Unfavorable Pilot-Vehicle Interactions. National Research Council, Washington, D.C. (1997)

2. Anon.: Safety management manual (SMM). ICAO Doc 9859, International Civil Aviation Organization, Second edition, (2009). ISBN 978-92-9231-265-4
3. Klyde, D.H., Mitchell, D.G: Investigating the role of rate limiting in pilot-induced oscillations. AIAA J. Guid. Control Dyn. 27(5):1 (2004). https://doi.org/10.2514/1.3215

4. Pavel, M., Jump, M., Dang-Vu, B., Masarati, P., Gennaretti, M., Ionita, A., Zaichik, L., Smaili, H., Quaranta, G., Yilmaz, D., Jones, M., Serafini, J., Malecki, J.: Adverse rotorcraft pilot couplings: past, present and future challenges. Prog. Aerosp. Sci. 62, $1-51(2013)$ cepts for takeover control using electronically coupled sidesticks.
5. Sampaio, R.S., Jones, M., Walko, C.: Evaluation of novel con- 
In: AHS 74th Annual Forum, AHS International, May 14-17., Phoenix AZ (2018)

6. Jones, M., Barnett, M.: Analysis of rotorcraft pilot couplings during active inceptor failures. In: AHS 74th Annual Forum, AHS International, May 14-17., Phoenix AZ (2018)

7. Klyde, D.H., Liang, C., Alvarez, D., Richards, N., Adams, R., Cogan, B.: Mitigating unfavorable pilot interactions with adaptive controllers in the presence of failures/damage. In: Proceedings of the AIAA Atmospheric Flight Mechanics Conference, 8-11 August. Portland, Oregon (2011)

8. Blanken, C., Lusardi, J., Ivler, C., Tischler, M., Hoefinger, M., Decker, W., Malpica, C., Berger, T., Tucker, G.: An investigation of rotorcraft stability-phase margin requirements in Hover. AHS 65th Annual Forum. AHS International, Grapevine, TX (2009)

9. Anon.: Aeronautical design standard performance specification handling qualities requirements for military rotorcraft. ADS-33EPRF, United States Army Aviation and Missile Command, Redstone Arsenal, AL (2000)

10. Tischler, M., Lee, J., Colbourne, J.: Comparison of flight control system design methods using the CONDUIT design tool. AIAA J. Guid. Control Dyn. 25, 482-493 (2002). https://doi. org/10.2514/2.4908

11. Duda, H.: Flight control system design considering rate saturation. Aerosp. Sci. Technol. 2(4), 1 (1998)

12. Tischler, M., Berger, T., Ivler, C., Mansur, M., Cheung, K., Soong, J.: Practical methods for aircraft and rotorcraft flight control design: an optimization-based approach. AIAA Education Series, ISBN:978-1-62410-443-5 (2017)

13. Dieterich, O., Pavel, M.: Rotorcraft-pilot coupling research in Europe. In: 5th CEAS Air and Space Conference. Challenges in European Aerospace, Delft, The Netherlands, 7-11 September (2015)

14. Dieterich, O., Goetz, J., Dang Vu, Binh., Haverdings, H., Masarati, P., Pavel, M., Jump, M., Gennaretti, M.: Adverse rotorcraft-pilot couplings: recent research activities in Europe. RAeS European Rotorcraft Forum, Liverpool, UK (2008)

15. Pavel, M., Jump, M., Masarati, P., Zaichik, L., Dang-Vu, B., Smaili, H., Quaranta, G., Stroosma, O., Yilmaz, D., Jones, M., Gennaretti, M., Ionita, A.: Practices to identify and prevent adverse aircraft and rotorcraft pilot couplings: a ground simulator perspective. Prog. Aerosp. Sci. 77, 54-87 (2015)

16. Mariano, V., Guglieri, G., Ragazzi, A.: Application of pilotinduced oscillation prediction to rotorcraft. In: Proceedings of the 37th European Rotorcraft Forum, September 13-15. Gallarate, Italy (2011)

17. Ossmann, D., Heller, M., Brieger, O.: Enhancement of the nonlinear OLOP-PIO-criterion regarding phase-compensated rate limiters. In: AIAA Atmosphic Flight Mechanics Conference and Exhibit, 18-21 August. Honolulu, Hawaii (2008)
18. Duda, H.: Fliegbarkeitskriterien bei begrenzter Stellgeschwindigkeit. Institute of Flight Mechanics, German Aerospace Center (1997 (in German)

19. Gilbreath, G.: Prediction of pilot-induced oscillations (PIO) due to actuator rate limiting using the open-loop onset point (OLOP) criteria. Masters Thesis, Air Force Institute of Technology, Wright Patterson Air Force Base, Ohio (2001)

20. Hess, R., Zeyada, Y., Heffley, R.: Modeling and simulation for helicopter task analysis. J. Am. Helicopter Soc. 47(4), 243-252 (2000)

21. Malpica, C., Lusardi, J.: Handling qualities analysis of active inceptor force-feel characteristics. In: AHS 69th Annual Forum, AHS International, 21-23 May., Phoenix AZ (2013)

22. McRuer, D.: Pilot-induced oscillations and human dynamic behaviour. NASA Contractor Report 4683, NASA (1995)

23. von Gruenhagen, W., Muelhaeuser, M., Hoefinger, M., Lusardi, J.: In-flight evaluation of active sidestick parameters for rate command and attitude command response types. AHS Handling Qualities Specialist Meeting, Huntsville, AL (2014)

24. Duda, H., Gerlach, T., Advani, S., Potter, M.: "Design of the DLR AVES Research Flight Simulator," Proceedings of the AIAA Modeling and Simulation Technologies Conference, 19-22 August. Massachusetts, Boston (2013)

25. Gotschlich, J., Jones, M.: Online trimming of flight dynamics models using the 2simulate realtime simulation framework. In: Proceedings of the AIAA Modeling and Simulation Technologies Conference, 8-12 Jan. Orlando, Florida (2018)

26. Nonnenmacher, D., Muellhaeuser, M.: Optimization of the equivalent mechanical characteristics of active side sticks for piloting a controlled helicopter. CEAS Aeronaut. J. 2(1-4), 157-170 (2011)

27. Jones, M., Jump, M.: New methods to subjectively and objectively evaluate adverse pilot couplings. J. Am. Helicopter Soc 60(1), 1-13 (2015). https://doi.org/10.4050/JAHS.60.011003

28. Jones, M., Jump, M., Lu, L.: Development of the phase-aggression criterion for rotorcraft pilot coupling detection. J. Guid. Control Dyn. 36(1), 35-47 (2013)

29. DiFranco, D.A.: Flight investigation of longitudinal short period frequency requirements and pio tendencies. Tech. Rep. AFFDLTR-66-163, Cornell Aeronautical Lab (1967)

30. Weingarten, N., Chalk, C.R.: In-flight investigation of large airplane flying qualities for approach and landing. Tech. Rep. AFWAL-TR-81-3118, Calspan (1981)

31. Jones, M.: Prediction, detection, and observation of rotorcraft pilot couplings. PhD Thesis, University of Liverpool, Liverpool (2014)

32. Mitchell, D.: Flight and ground testing for pilot-induced oscillations. In: IEEE Aerospace Applications Conference Proceedings, Snowmass at Aspen, CO (1999) 